\title{
Links of complex analytic singularities
}

\author{
János Kollár
}

Let $X$ be a complex algebraic or analytic variety. Its local topology near a point $x \in X$ is completely described by its link $L(x \in X)$, which is obtained as the intersection of $X$ with a sphere of radius $0<\epsilon \ll 1$ centered at $x$. The intersection of $X$ with the closed ball of radius $\epsilon$ centered at $x$ is homeomorphic to the cone over $L(x \in X)$; cf. [GM88, p.41].

If $x \in X$ is a smooth point then its link is a sphere of dimension $2 \operatorname{dim}_{\mathbb{C}} X-1$. Conversely, if $X$ is a normal surface and $L(x \in X)$ is a sphere then $x$ is a smooth point [Mum61], but this fails in higher dimensions [Bri66].

The aim of this survey is to study in some sense the opposite question: we are interested in the "most complicated" links. In its general form, the question is the following.

PROBLEM 1. Which topological spaces can be links of complex algebraic or analytic singularities?

If $\operatorname{dim} X=1$, then the possible links are disjoint unions of circles. The answer is much more complicated in higher dimensions and we focus on isolated singularities from now on, though many results hold for non-isolated singularities as well. Thus the link $L(x \in X)$ is a (differentiable) manifold of (real) dimension $2 \operatorname{dim}_{\mathbb{C}} X-1$.

Among the simplest singularities are the cones over smooth projective varieties. Let $Z \subset \mathbb{P}^{N}$ be a smooth projective variety and $X:=\operatorname{Cone}(Z) \subset$ $\mathbb{C}^{N+1}$ the cone over $Z$ with vertex at the origin. Then $L(0 \in X)$ is a circle bundle over $Z$ whose first Chern class is the hyperplane class. Thus the link of the vertex of Cone $(Z)$ is completely described by the base $Z$ and by the hyperplane class $[H] \in H^{2}(Z, \mathbb{Z})$.

Note that a singularity $0 \in X \subset \mathbb{C}^{N}$ is a cone iff it can be defined by homogeneous equations. One gets a much larger class of singularities if we consider homogeneous equations where different variables have different degree (or weight).

For a long time it was believed that links of isolated singularities are "very similar" to links of cones and weighted cones. The best illustration of 
this is given by the complete description of links of surface singularities given in [Neu81]. Cones give circle bundles over Riemann surfaces and weighted cones give Seifert bundles over Riemann surfaces. General links are more complicated but they are all obtained by gluing Seifert bundles over Riemann surfaces with boundary. These are definitely more complicated than Seifert bundles, but much simpler than general 3-manifolds. In particular, hyperbolic 3-manifolds - which comprise the largest and most complicated class - do not occur as links.

Important examples of the similarity of general links to smooth projective varieties are given by the local Lefschetz theorems, initiated by Grothendieck [Gro68] and developed much further subsequently; see [GM88] for a detailed treatment.

As another illustration, the weights of the mixed Hodge structure on the cohomology groups of links also follow the same pattern for general links as for links of cones, see [DH88] or [PS08, Sec.6.3].

These and many other examples led to a viewpoint that was best summarized in [GM88, p.26]: "Philosophically, any statement about the projective variety or its embedding really comes from a statement about the singularity at the point of the cone. Theorems about projective varieties should be consequences of more general theorems about singularities which are no longer required to be conical."

Recently this belief was called into question by [KK11] which proved that fundamental groups of general links are very different from fundamental groups of links of cones. The aim of this paper is to summarize the results, present several new theorems and review the problems that arise.

Philosophically, the main long term question is to understand the limits of the above principle. We know that it fails for the fundamental group but it seems to apply to cohomology groups. It is unclear if it applies to simply connected links or not.

The new results rely on a method, considered in [Kol11], to construct singularities using their resolution. By Hironaka's resolution theorem, for every isolated singularity $(x \in X)$ there is a proper, birational morphism $f: Y \rightarrow X$ such that $E:=f^{-1}(x)$ is a simple normal crossing divisor and $Y \backslash E \rightarrow X \backslash\{x\}$ is an isomorphism. The method essentially reverses the resolution process. That is, we start with a (usually reducible) simple normal crossing variety $E$, embed $E$ into a smooth variety $Y$ and then contract $E \subset Y$ to a point to obtain $(x \in X)$. If $E$ is smooth, this is essentially the cone construction.

This approach has been one of the standard ways to construct surface singularities but it has not been investigated in higher dimensions until recently. There were probably two reason for this. First, if $\operatorname{dim} X \geq 3$ then there is no "optimal" choice for the resolution $f: Y \rightarrow X$. Thus the exceptional set $E=f^{-1}(x)$ depends on many arbitrary choices and it is not easy to extract any invariant of the singularity from $E$; see, however, Definition 6 . Thus any construction starting with $E$ seemed rather arbitrary. 
Second, the above philosophy suggested that one should not get anything substantially new this way.

The first indication that this method is worth exploring was given in [Kol11] where it was used to construct new examples of terminal and log canonical singularities that contradicted earlier expectations.

A much more significant application was given in [KK11]. Since in higher dimensions a full answer to Problem 1 may well be impossible to give, it is sensible to focus on some special aspects. A very interesting question turned out to be the following.

PROBLEM 2. Which groups occur as fundamental groups of links of complex algebraic or analytic singularities?

Note that the fundamental groups of smooth projective varieties are rather special; see $\left[\mathbf{A B C}^{+} \mathbf{9 6}\right]$ for a survey. Even the fundamental groups of smooth quasi projective varieties are quite restricted [Mor78, KM98a, CS08, DPS09]. By contrast fundamental groups of links are arbitrary.

TheOREM 3. [KK11] For every finitely presented group $G$ there is an isolated, complex singularity $\left(0 \in X_{G}\right)$ with link $L_{G}$ such that $\pi_{1}\left(L_{G}\right) \cong G$.

Note that once such a singularity exists, a local Lefschetz-type theorem (cf. [GM88, Sec.II.1.2]) implies that the link of a general 3-dimensional hyperplane section has the same fundamental group.

There are two natural directions to further develop this result: one can connect properties of the fundamental group of a link to algebraic or analytic properties of a singularity and one can investigate further the topology of the links or of the resolutions.

In the first direction, the following result answers a question of Wahl.

TheOREm 4. For a finitely presented group $G$ the following are equivalent.

(1) $G$ is $\mathbb{Q}$-perfect, that is, its largest abelian quotient is finite.

(2) $G$ is the fundamental group of the link of an isolated CohenMacaulay singularity (46) of dimension $\geq 3$.

One can study the local topology of $X$ by choosing a resolution of singularities $\pi: Y \rightarrow X$ such that $E_{x}:=\pi^{-1}(x) \subset Y$ is a simple normal crossing divisor and then relating the topology of $E_{x}$ to the topology of the link $L(x \in X)$.

The topology of a simple normal crossing divisor $E$ can in turn be understood in 2 steps. First, the $E_{i}$ are smooth projective varieties, and their topology is much studied. A second layer of complexity comes from how the components $E_{i}$ are glued together. This gluing process can be naturally encoded by a finite cell complex $\mathcal{D}(E)$, called the dual complex or dual graph of $E$. 
Definition 5 (Dual complex). Let $E$ be a variety with irreducible components $\left\{E_{i}: i \in I\right\}$. We say that $E$ is a simple normal crossing variety (abbreviated as snc) if the $E_{i}$ are smooth and every point $p \in E$ has an open (Euclidean) neighborhood $p \in U_{p} \subset E$ and an embedding $U_{p} \hookrightarrow \mathbb{C}^{n+1}$ such that the image of $U_{p}$ is an open subset of the union of coordinate hyperplanes $\left(z_{1} \cdots z_{n+1}=0\right)$. A stratum of $E$ is any irreducible component of an intersection $\cap_{i \in J} E_{i}$ for some $J \subset I$.

The combinatorics of $E$ is encoded by a cell complex $\mathcal{D}(E)$ whose vertices are labeled by the irreducible components of $E$ and for every stratum $W \subset \cap_{i \in J} E_{i}$ we attach a $(|J|-1)$-dimensional cell. Note that for any $j \in J$ there is a unique irreducible component of $\cap_{i \in J \backslash\{j\}} E_{i}$ that contains $W$; this specifies the attaching map. $\mathcal{D}(E)$ is called the dual complex or dual graph of $E$. (Although $\mathcal{D}(E)$ is not a simplicial complex in general, it is an unordered $\Delta$-complex in the terminology of [Hat02, p.534].)

Definition 6 (Dual complexes associated to a singularity). Let $X$ be a normal variety and $x \in X$ a point. Choose a resolution of singularities $\pi: Y \rightarrow X$ such that $E_{x}:=\pi^{-1}(x) \subset Y$ is a simple normal crossing divisor. Thus it has a dual complex $\mathcal{D}\left(E_{x}\right)$.

The dual graph of a normal surface singularity has a long history. Higher dimensional versions appear in [Kul77, Per77, Gor80, FM83] but systematic investigations were started only recently; see [Thu07, Ste08, Pay09, Pay11].

It is proved in [Thu07, Ste08, ABW11] that the homotopy type of $\mathcal{D}\left(E_{x}\right)$ is independent of the resolution $Y \rightarrow X$. We denote it by $\mathcal{D} \mathcal{R}(x \in X)$.

The proof of Theorem 3 gives singularities for which the fundamental group of the link is isomorphic to the fundamental group of $\mathcal{D} \mathcal{R}(x \in X)$. In general, it seems easier to study $\mathcal{D} \mathcal{R}(x \in X)$ than the link and the next theorem shows that not just the fundamental group but the whole homotopy type of $\mathcal{D} \mathcal{R}(0 \in X)$ can be arbitrary. The additional properties $(7.2-3)$ follow from the construction as in [Kol11, KK11].

TheOREM 7. Let $T$ be a connected, finite cell complex. Then there is a normal singularity $(0 \in X)$ such that

(1) the complex $\mathcal{D} \mathcal{R}(0 \in X)$ is homotopy equivalent to $T$,

(2) $\pi_{1}(L(0 \in X)) \cong \pi_{1}(T)$ and

(3) if $\pi: Y \rightarrow X$ is any resolution then $R^{i} \pi_{*} \mathcal{O}_{Y} \cong H^{i}(T, \mathbb{C})$ for $i>0$.

The fundamental groups of the dual complexes of rational singularities (52) were determined in [KK11, Thm.42]. The next result extends this by determining the possible homotopy types of $\mathcal{D} \mathcal{R}(0 \in X)$.

THEOREM 8. Let $T$ be a connected, finite cell complex. Then there is a rational singularity $(0 \in X)$ whose dual complex $\mathcal{D} \mathcal{R}(0 \in X)$ is homotopy equivalent to $T$ iff $T$ is $\mathbb{Q}$-acyclic, that is, $H^{i}(T, \mathbb{Q})=0$ for $i>0$.

As noted in [Pay11], the dual complex $\mathcal{D} \mathcal{R}(0 \in X)$ can be defined even up-to simple-homotopy equivalence [Coh73]. The proofs given in 
[KK11] use Theorem 25, which in turn relies on some general theorems of [Cai61, Hir62] that do not seem to give simple-homotopy equivalence. ${ }^{1}$

\section{Content of the Sections.}

Cones, weighted cones and the topology of the corresponding links are discussed in Section 1.

The plan for the construction of singularities from their resolutions is outlined in Section 2 and the rest of the paper essentially fleshes out the details.

In Section 3 we show that every finite cell complex is homotopy equivalent to a Voronoi complex. These Voronoi complexes are then used to construct simple normal crossing varieties in Section 4.

The corresponding singularities are constructed in Section 5 where we prove Theorem 7 except for an explicit resolution of the resulting singularities which is accomplished in Section 6.

The proof of Theorem 4 is given in Section 7 where several other equivalent conditions are also treated. Theorem 8 on rational singularities is reviewed in Section 8.

Open questions and problems are discussed in Section 9.

Acknowledgments. I thank I. Dolgachev, T. de Fernex, T. Jarvis, M. Kapovich, L. Maxim, A. Némethi, P. Ozsváth, S. Payne, P. PopescuPampu, M. Ramachandran, J. Shaneson, T. Szamuely, D. Toledo, B. Totaro, J. Wahl, and C. Xu for comments and corrections. Partial financial support was provided by the NSF under grant number DMS-07-58275 and by the Simons Foundation. Part of this paper was written while the author visited the University of Utah.

\section{Weighted homogeneous links}

Definition 9 (Weighted homogeneous singularities). Assign positive weights to the variables $w\left(x_{i}\right) \in \mathbb{Z}$, then the weight of a monomial $\prod_{i} x_{i}^{a_{i}}$ is

$$
w\left(\prod_{i} x_{i}^{a_{i}}\right):=\sum_{i} a_{i} w\left(x_{i}\right) .
$$

A polynomial $f$ is called weighted homogeneous of weighted-degree $w(f)$ iff every monomial that occurs in $f$ with nonzero coefficient has weight $w(f)$.

Fix weights $\mathbf{w}:=\left(w\left(x_{1}\right), \ldots, w\left(x_{N}\right)\right)$ and let $\left\{f_{i}: i \in I\right\}$ be weighted homogeneous polynomials. They define both a projective variety in a weighted projective space

$$
Z\left(f_{i}: i \in I\right) \subset \mathbb{P}(\mathbf{w})
$$

and an affine weighted cone

$$
C\left(f_{i}: i \in I\right) \subset \mathbb{C}^{N} .
$$

Somewhat loosely speaking, a singularity is called weighted homogeneous if it is isomorphic to a singularity defined by a weighted cone for some weights

\footnotetext{
${ }^{1}$ This problem is settled in $[\mathbf{K o l 1 3 a}]$.
} 
$w\left(x_{i}\right)$. (In the literature these are frequently called quasi-homogeneous singularities.)

In many cases the weights are uniquely determined by the singularity (up to rescaling) but not always. For instance, the singularity $\left(x y=z^{n}\right)$ is weighted homogeneous for any weights that satisfy $w(x)+w(y)=n \cdot w(z)$.

If $C \subset \mathbb{C}^{N}$ is a weighted cone then it has a $\mathbb{C}^{*}$-action given by

$$
\left(x_{1}, \ldots, x_{N}\right) \mapsto\left(t^{m_{1}} x_{1}, \ldots, t^{m_{N}} x_{N}\right) \quad \text { where } \quad m_{i}=\frac{1}{w\left(x_{i}\right)} \prod_{j} w\left(x_{j}\right) .
$$

Conversely, let $X$ be a variety with a $\mathbb{C}^{*}$ action and $x \in X$ a fixed point that is attractive as $t \rightarrow 0$. Linearizing the action shows that $x \in X$ is a weighted homogeneous singularity.

10 (Links of weighted homogeneous singularities). The $\mathbb{C}^{*}$-action on a weighted homogeneous singularity $(x \in X)$ induces a fixed point free $\mathbb{S}^{1}$-action on its link $L$. If we think of $X$ as a weighted cone over the corresponding projective variety $Z \subset \mathbb{P}(\mathbf{w})$ then we get a projection $\pi$ : $L \rightarrow Z$ whose fibers are exactly the orbits of the $\mathbb{S}^{1}$-action, that is, the link of a weighted homogeneous singularity has a Seifert bundle structure. (For our purposes we can think that a Seifert bundle is the same as a fixed point free $\mathbb{S}^{1}$-action.) If $(x \in X)$ is an isolated singularity then $Z$ is an orbifold.

It is thus natural to study the topology of links of weighted homogeneous singularities in two steps.

(1) Describe all $2 n-1$-manifolds with a fixed point free $\mathbb{S}^{1}$-action.

(2) Describe which among them occur as links of weighted homogeneous singularities.

11 (Homology of a weighted homogeneous link). [OW75] Let $\pi: L \rightarrow Z$ be the Seifert bundle structure. The cohomology of $L$ is computed by a spectral sequence

$$
H^{i}\left(Z, R^{j} \pi_{*} \mathbb{Q}_{L}\right) \Rightarrow H^{i+j}(L, \mathbb{Q}) .
$$

All the fibers are oriented circles, thus $R^{0} \pi_{*} \mathbb{Q}_{L} \cong R^{1} \pi_{*} \mathbb{Q}_{L} \cong \mathbb{Q}_{Z}$ and $R^{j} \pi_{*} \mathbb{Q}_{L}=0$ for $j>1$. Thus the $E_{2}$-term of the spectral sequence is

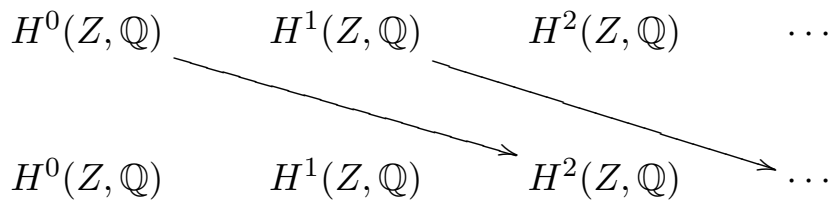

where the differentials are cup product with the (weighted) hyperplane class

$$
c_{1}\left(\mathcal{O}_{Z}(1)\right) \cup: H^{i}\left(Z, R^{1} \pi_{*} \mathbb{Q}_{L}\right) \cong H^{i}(Z, \mathbb{Q}) \rightarrow H^{i+2}(Z, \mathbb{Q}) .
$$

Since $Z$ is an orbifold, these are injective if $i+2 \leq \operatorname{dim} Z$ and surjective if $i \geq \operatorname{dim} Z$. Thus we conclude that

$$
\begin{array}{lll}
h^{i}(L, \mathbb{Q}) & =h^{i}(Z, \mathbb{Q})-h^{i-2}(Z, \mathbb{Q}) & \text { if } i \leq \operatorname{dim} Z \text { and } \\
h^{i+1}(L, \mathbb{Q}) & =h^{i}(Z, \mathbb{Q})-h^{i+2}(Z, \mathbb{Q}) & \text { if } i \geq \operatorname{dim} Z
\end{array}
$$


where we set $h^{i}(Z, \mathbb{Q})=0$ for $i<0$ or $i>2 \operatorname{dim} Z$. In particular we see that $L$ is a rational homology sphere iff $Z$ is a rational homology complex projective space.

By contrast, the spectral sequence computing the integral cohomology of $L$ is much more complicated. We have a natural injection $R^{1} \pi_{*} \mathbb{Z}_{L} \hookrightarrow \mathbb{Z}_{Z}$ which is, however, rarely an isomorphism. The computations were carried out only for $\operatorname{dim} L \leq 5$ [Kol05].

12 (Weighted homogeneous surface singularities). This is the only case that is fully understood.

The classification of fixed point free circle actions on 3-manifolds was considered by Seifert [Sei32]. If $M$ is a 3-manifold with a fixed point free circle action then the quotient space $F:=M / \mathbb{S}^{1}$ is a surface (without boundary in the orientable case). The classification of these Seifert fibered 3 -manifolds $f: M \rightarrow F$ is thus equivalent to the classification of fixed point free circle actions. It should be noted that already in this classical case, it is conceptually better to view the base surface $F$ not as a $2-$ manifold but as a 2-dimensional orbifold, see [Sco83] for a detailed survey from this point of view.

Descriptions of weighted homogeneous surface singularities are given in [Pin77, Dol83, Dem88, FZ03].

\section{Weighted homogeneous 3-fold singularities.}

There is a quite clear picture about the simply connected case since simply connected 5 -manifolds are determined by their homology.

By a theorem of [Sma62, Bar65], a simply connected, compact 5manifold $L$ is uniquely determined by $H_{2}(L, \mathbb{Z})$ and the second StiefelWhitney class, which we view as a map $w_{2}: H_{2}(L, \mathbb{Z}) \rightarrow \mathbb{Z} / 2$. Furthermore, there is such a 5 -manifold iff there is an integer $k \geq 0$ and a finite Abelian group $A$ such that either $H_{2}(L, \mathbb{Z}) \cong \mathbb{Z}^{k}+A+A$ and $w_{2}: H_{2}(L, \mathbb{Z}) \rightarrow \mathbb{Z} / 2$ is arbitrary, or $H_{2}(L, \mathbb{Z}) \cong \mathbb{Z}^{k}+A+A+\mathbb{Z} / 2$ and $w_{2}$ is projection on the $\mathbb{Z} / 2$-summand.

The existence of Seifert bundles on simply connected compact 5manifolds was treated in [Kol06]. The answer mostly depends on the torsion subgroup of $H_{2}(L, \mathbb{Z})$, but there is a subtle interplay with $w_{2}$.

Definition 13. Let $M$ be any manifold. Write its second homology as a direct sum of cyclic groups of prime power order

$$
H_{2}(M, \mathbb{Z})=\mathbb{Z}^{k}+\sum_{p, i}\left(\mathbb{Z} / p^{i} \mathbb{Z}\right)^{c\left(p^{i}\right)}
$$

for some $k=\operatorname{dim} H_{2}(M, \mathbb{Q})$ and $c\left(p^{i}\right)=c\left(p^{i}, M\right)$. The numbers $k, c\left(p^{i}\right)$ are determined by $H_{2}(M, \mathbb{Z})$ but the subgroups $\left(\mathbb{Z} / p^{i}\right)^{c\left(p^{i}\right)} \subset H_{2}(M, \mathbb{Z})$ are usually not unique. One can choose the decomposition (13.1) such that $w_{2}: H_{2}(M, \mathbb{Z}) \rightarrow \mathbb{Z} / 2$ is zero on all but one summand $\mathbb{Z} / 2^{n}$. This value $n$ is unique and it is denoted by $i(M)$ [Bar65]. This invariant can take up any value $n$ for which $c\left(2^{n}\right) \neq 0$, besides 0 and $\infty$. Alternatively, $i(M)$ is the 
smallest $n$ such that there is an $\alpha \in H_{2}(M, \mathbb{Z})$ such that $w_{2}(\alpha) \neq 0$ and $\alpha$ has order $2^{n}$.

The existence of a fixed point free differentiable circle action puts strong restrictions on $\mathrm{H}_{2}$ and on $w_{2}$.

Theorem 14. [Kol06, Thm.3] Let L be a compact, simply connected 5-manifold. Then $L$ admits a fixed point free differentiable circle action if and only if $H_{2}(L, \mathbb{Z})$ and $w_{2}$ satisfy the following conditions.

(1) For every $p$, we have at most $\operatorname{dim} H_{2}(M, \mathbb{Q})+1$ nonzero $c\left(p^{i}\right)$ in (13.1).

(2) One can arrange that $w_{2}: H_{2}(L, \mathbb{Z}) \rightarrow \mathbb{Z} / 2$ is the zero map on all but the $\mathbb{Z}^{k}+(\mathbb{Z} / 2)^{c(2)}$ summands in (13.1). That is, $i(L) \in$ $\{0,1, \infty\}$.

(3) If $i(L)=\infty$ then $\#\left\{i: c\left(2^{i}\right)>0\right\} \leq \operatorname{dim} H_{2}(M, \mathbb{Q})$.

REMARK 15. Note that while Theorem 14 tells us which compact, simply connected 5-manifolds admit a fixed point free differentiable circle action, the proof does not classify all circle actions. In particular, the classification of all circle actions on $\mathbb{S}^{5}$ is not known.

By contrast very little is known about which compact, simply connected 5 -manifolds occur as links of weighted homogeneous singularities. It is known that not every Seifert bundle occurs [Kol06, Lem.49] but a full answer seems unlikely.

Nothing seems to be known in higher dimensions.

16 (Einstein metrics on weighted homogeneous links). By a result of [Kob63], the link of a cone over a smooth projective variety $Z \subset \mathbb{P}^{N}$ carries a natural Einstein metric iff $-K_{Z}$ is a positive multiple of the hyperplane class and $Z$ carries a Kähler-Einstein metric. This was generalized by [BG00] to weighted cones. Here one needs to work with an orbifold canonical class $K_{X}+\Delta$ and a suitable orbifold Kähler-Einstein metric on $(X, \Delta)$.

This approach was used to construct new Einstein metrics on spheres and exotic spheres [BGK05, BGKT05] and on many 5-manifolds [Kol05, Kol07a, Kol09].

See [BG08] for a comprehensive treatment.

\section{Construction of singularities}

The construction has 5 main steps, none of which is fully understood at the moment. After summarizing them, we discuss the difficulties in more detail. Although the steps can not be carried out in full generality, we understand enough about them to obtain the main theorems.

17 (Main steps of the construction).

Step.17.1. For a simplicial complex $C$ construct projective simple normal crossing varieties $V(C)$ such that $\mathcal{D}(V(C)) \cong C$. 
Step.17.2. For a projective simple normal crossing variety $V$ construct a smooth variety $Y(V)$ that contains $V$ as a divisor.

Step.17.3. For a smooth variety $Y$ containing a simple normal crossing divisor $D$ construct an isolated singularity $(x \in X)$ such that $(D \subset Y)$ is a resolution of $(x \in X)$.

Step.17.4. Describe the link $L(x \in X)$ in terms of the topology of $D$ and the Chern class of the normal bundle of $D$.

Step.17.5. Describe the relationship between the properties of the singularity $(x \in X)$ and the original simplicial complex $C$.

18 (Discussion of Step 17.1). I believe that for every simplicial complex $C$ there are many projective simple normal crossing varieties $V(C)$ such that $\mathcal{D}(V(C)) \cong C .^{2}$

There seem to be two main difficulties of a step-by-step approach.

First, topology would suggest that one should build up the skeleta of $V(\mathcal{C})$ one dimension at a time. It is easy to obtain the 1 -skeleton by gluing rational curves. The 2 -skeleton is still straightforward since rational surfaces do contain cycles of rational curves of arbitrary length. However, at the next step we run into a problem similar to Step 17.2 and usually a 2-skeleton can not be extended to a 3 -skeleton. Our solution in $[\mathbf{K K 1 1}]$ is to work with triangulations of $n$-dimensional submanifolds with boundary in $\mathbb{R}^{n}$. The ambient $\mathbb{R}^{n}$ gives a rigidification and this makes it possible to have a consistent choice for all the strata.

Second, even if we construct a simple normal crossing variety $V$, it is not easy to decide whether it is projective. This is illustrated by the following example of "triangular pillows" [KK11, Exmp.34].

Let us start with an example that is not simple normal crossing.

Take 2 copies $\mathbb{P}_{i}^{2}:=\mathbb{P}^{2}\left(x_{i}: y_{i}: z_{i}\right)$ of $\mathbb{C P}^{2}$ and the triangles $C_{i}:=$ $\left(x_{i} y_{i} z_{i}=0\right) \subset \mathbb{P}_{i}^{2}$. Given $c_{x}, c_{y}, c_{z} \in \mathbb{C}^{*}$, define $\phi\left(c_{x}, c_{y}, c_{z}\right): C_{1} \rightarrow C_{2}$ by $\left(0: y_{1}: z_{1}\right) \mapsto\left(0: y_{1}: c_{z} z_{1}\right),\left(x_{1}: 0: z_{1}\right) \mapsto\left(c_{x} x_{1}: 0: z_{1}\right)$ and $\left(x_{1}: y_{1}: 0\right) \mapsto\left(x_{1}: c_{y} y_{1}: 0\right)$ and glue the 2 copies of $\mathbb{P}^{2}$ using $\phi\left(c_{x}, c_{y}, c_{z}\right)$ to get the surface $S\left(c_{x}, c_{y}, c_{z}\right)$.

We claim that $S\left(c_{x}, c_{y}, c_{z}\right)$ is projective iff the product $c_{x} c_{y} c_{z}$ is a root of unity.

To see this note that $\operatorname{Pic}^{0}\left(C_{i}\right) \cong \mathbb{C}^{*}$ and $\operatorname{Pic}^{r}\left(C_{i}\right)$ is a principal homogeneous space under $\mathbb{C}^{*}$ for every $r \in \mathbb{Z}$. We can identify $\operatorname{Pic}^{3}\left(C_{i}\right)$ with $\mathbb{C}^{*}$ using the restriction of the ample generator $L_{i}$ of $\operatorname{Pic}\left(\mathbb{P}_{i}^{2}\right) \cong \mathbb{Z}$ as the base point.

The key observation is that $\phi\left(c_{x}, c_{y}, c_{z}\right)^{*}: \operatorname{Pic}^{3}\left(C_{2}\right) \rightarrow \operatorname{Pic}^{3}\left(C_{1}\right)$ is multiplication by $c_{x} c_{y} c_{z}$. Thus if $c_{x} c_{y} c_{z}$ is an $r$ th root of unity then $L_{1}^{r}$ and $L_{2}^{r}$ glue together to an ample line bundle but otherwise $S\left(c_{x}, c_{y}, c_{z}\right)$ carries only the trivial line bundle.

\footnotetext{
${ }^{2}$ This is now proved in [Kol13a].
} 
We can create a similar simple normal crossing example by smoothing the triangles $C_{i}$. That is, we take 2 copies $\mathbb{P}_{i}^{2}:=\mathbb{P}^{2}\left(x_{i}: y_{i}: z_{i}\right)$ of $\mathbb{C P}^{2}$ and smooth elliptic curves $E_{i}:=\left(x_{i}^{3}+y_{i}^{3}+z_{i}^{3}=0\right) \subset \mathbb{P}_{i}^{2}$.

Every automorphism $\tau \in \operatorname{Aut}\left(x^{3}+y^{3}+z^{3}=0\right)$ can be identified with an isomorphism $\tau: E_{1} \cong E_{2}$, giving a simple normal crossing surface $S(\tau)$. The above argument then shows that $S(\tau)$ is projective iff $\tau^{m}=1$ for some $m>0$.

These examples are actually not surprising. One can think of the surfaces $S\left(c_{x}, c_{y}, c_{z}\right)$ and $S(\tau)$ as degenerate K3 surfaces of degree 2 and K3 surfaces have non-projective deformations. Similarly, $S\left(c_{x}, c_{y}, c_{z}\right)$ and $S(\tau)$ can be non-projective. One somewhat unusual aspect is that while a smooth K3 surface is projective iff it is a scheme, the above singular examples are always schemes yet many of them are non-projective.

19 (Discussion of Step 17.2). This is surprisingly subtle. First note that not every projective simple normal crossing variety $V$ can be realized as a divisor on a smooth variety $Y$. A simple obstruction is the following.

Let $Y$ be a smooth variety and $D_{1}+D_{2}$ a simple normal crossing divisor on $Y$. Set $Z:=D_{1} \cap D_{2}$. Then $\left.N_{Z, D_{2}} \cong N_{D_{1}, Y}\right|_{Z}$ where $N_{X, Y}$ denotes the normal bundle of $X \subset Y$.

Thus if $V=V_{1} \cup V_{2}$ is a simple normal crossing variety with $W:=V_{1} \cap V_{2}$ such that $N_{W, V_{2}}$ is not the restriction of any line bundle from $V_{1}$ then $V$ is not a simple normal crossing divisor in a nonsingular variety.

I originally hoped that such normal bundle considerations give necessary and sufficient conditions, but recent examples of [Fuj12a, Fuj12b] show that this is not the case.

For now, no necessary and sufficient conditions of embeddability are known. In the original papers [Kol11, KK11] we went around this problem by first embedding a simple normal crossing variety $V$ into a singular variety $Y$ and then showing that for the purposes of computing the fundamental group of the link the singularities of $Y$ do not matter.

We improve on this in Section 6.

20 (Discussion of Step 17.3). By a result of [Art70], a compact divisor contained in a smooth variety $D=\cup_{i} D_{i} \subset Y$ can be contracted to a point if there are positive integers $m_{i}$ such that $\left.\mathcal{O}_{Y}\left(-\sum_{i} m_{i} D_{i}\right)\right|_{D_{j}}$ is ample for every $j$.

It is known that this condition is not necessary and no necessary and sufficient characterizations are known. However, it is easy to check the above condition in our examples.

21 (Discussion of Step 17.4). This approach, initiated in [Mum61], has been especially successful for surfaces.

In principle the method of [Mum61] leads to a complete description of the link, but it seems rather difficult to perform explicit computations. Computing the fundamental group of the links seems rather daunting in general. Fortunately, we managed to find some simple conditions that ensure 
that the natural maps

$$
\pi_{1}(L(x \in X)) \rightarrow \pi_{1}(\mathcal{R}(X)) \rightarrow \pi_{1}(\mathcal{D} \mathcal{R}(X))
$$

are isomorphisms. However, these simple conditions force $D$ to be more complicated than necessary, in particular we seem to lose control of the canonical class of $X$.

22 (Discussion of Step 17.5). For surfaces there is a very tight connection between the topology of the link and the algebro-geometric properties of a singularity. In higher dimension, one can obtain very little information from the topology alone. As we noted, there are many examples where $X$ is a topological manifold yet very singular as a variety.

There is more reason to believe that algebro-geometric properties restrict the topology. For example, the results of Section 7 rely on the observation that if $(x \in X)$ is a rational (or even just 1-rational) singularity then $H_{1}(L(x \in X), \mathbb{Q})=0$.

\section{Voronoi complexes}

Definition 23. A (convex) Euclidean polyhedron is a subset $P$ of $\mathbb{R}^{n}$ given by a finite collection of linear inequalities (some of which may be strict and some not). A face of $P$ is a subset of $P$ which is given by converting some of these non-strict inequalities to equalities.

A Euclidean polyhedral complex in $\mathbb{R}^{n}$ is a collection of closed Euclidean polyhedra $\mathcal{C}$ in $\mathbb{R}^{n}$ such that

(1) if $P \in \mathcal{C}$ then every face of $P$ is in $\mathcal{C}$ and

(2) if $P_{1}, P_{2} \in \mathcal{C}$ then $P_{1} \cap P_{2}$ is a face of both of the $P_{i}$ (or empty).

The union of the faces of a Euclidean polyhedral complex $\mathcal{C}$ is denoted by $|\mathcal{C}|$.

For us the most important examples are the following.

Definition 24 (Voronoi complex). Let $Y=\left\{y_{i}: i \in I\right\} \subset \mathbb{R}^{n}$ be a finite subset. For each $i \in I$ the corresponding Voronoi cell is the set of points that are closer to $y_{i}$ than to any other $y_{j}$, that is

$$
V_{i}:=\left\{x \in \mathbb{R}^{n}: d\left(x, y_{i}\right) \leq d\left(x, y_{j}\right), \forall j \in I\right\}
$$

where $d(x, y)$ denotes the Euclidean distance. Each cell $V_{i}$ is a closed (possibly unbounded) polyhedron in $\mathbb{R}^{n}$.

The Voronoi cells and their faces give a Euclidean polyhedral complex, called the Voronoi complex or Voronoi tessellation associated to $Y$.

For a subset $J \subset I$ let $H_{J}$ denote the linear subspace

$$
H_{J}:=\left\{x \in \mathbb{R}^{n}: d\left(x, y_{i}\right)=d\left(x, y_{j}\right) \forall i, j \in J\right\} .
$$

The affine span of each face of the Voronoi complex is one of the $H_{J}$. If $J$ has 2 elements $\{i, j\}$ then $H_{i j}$ is a hyperplane $H_{i j}=\left\{x \in \mathbb{R}^{n}: d\left(x, y_{i}\right)=\right.$ $\left.d\left(x, y_{j}\right)\right\}$. 
A Voronoi complex is called simple if for every $k$, every codimension $k$ face is contained in exactly $k+1$ Voronoi cells. Not every Voronoi complex is simple, but it is easy to see that among finite subsets $Y \subset \mathbb{R}^{n}$ those with a simple Voronoi complex $\mathcal{C}(Y)$ form an open and dense set.

Let $\mathcal{C}$ be a simple Voronoi complex. For each face $F \in \mathcal{C}$, let $V_{i}$ for $i \in I_{F}$ be the Voronoi cells containing $F$. The vertices $\left\{y_{i}: i \in I_{F}\right\}$ form a simplex whose dimension equals the codimension of $F$. These simplices define the Delaunay triangulation dual to $\mathcal{C}$.

TheOREM 25. [KK11, Cor.21] Let $T$ be a finite simplicial complex of dimension $n$. Then there is an embedding $j: T \hookrightarrow \mathbb{R}^{2 n+1}$, a simple Voronoi complex $\mathcal{C}$ in $\mathbb{R}^{2 n+1}$ and a subcomplex $\mathcal{C}(T) \subset \mathcal{C}$ of pure dimension $2 n+1$ containing $j(T)$ such that the inclusion $j(T) \subset|\mathcal{C}(T)|$ is a homotopy equivalence.

Outline of the proof. First we embed $T$ into $\mathbb{R}^{2 n+1}$. This is where the dimension increase comes from. (We do not need an actual embedding, only an embedding up-to homotopy, which is usually easier to get.)

Then we first use a result of [Hir62] which says that if $T$ is a finite simplicial complex in a smooth manifold $\mathbf{R}$ then there exists a codimension 0 compact submanifold $M \subset \mathbf{R}$ with smooth boundary containing $T$ such that the inclusion $T \subset M$ is a homotopy equivalence.

Finally we construct a Voronoi complex using $M$.

Let $M \subset \mathbb{R}^{m}$ be a compact subset, $Y \subset \mathbb{R}^{m}$ a finite set of points and $\mathcal{C}(Y)$ the corresponding Voronoi complex. Let $\mathcal{C}_{m}(Y, M)$ be the collection of those $m$-cells in the Voronoi complex $\mathcal{C}(Y)$ whose intersection with $M$ is not empty and $\mathcal{C}(Y, M)$ the polyhedral complex consisting of the cells in $\mathcal{C}_{m}(Y, M)$ and their faces. Then $M \subset|\mathcal{C}(Y, M)|$.

We conclude by using a theorem of [Cai61] that says that if $M$ is a $C^{2}$-submanifold with $C^{2}$-boundary then for a suitably fine mesh of points $Y \subset \mathbb{R}^{m}$ the inclusion $M \subset|\mathcal{C}(Y, M)|$ is a homotopy equivalence.

\section{Simple normal crossing varieties}

Let $\mathcal{C}$ be a purely $m$-dimensional, compact subcomplex of a simple Voronoi complex in $\mathbb{R}^{m}$. Our aim is to construct a projective simple normal crossing variety $V(\mathcal{C})$ whose dual complex naturally identifies with the Delaunay triangulation of $\mathcal{C}$.

26 (First attempt). For each $m$-polytope $P_{i} \in \mathcal{C}$ we associate a copy $\mathbb{P}_{(i)}^{m}=\mathbb{C P}^{m}$. For a subvariety $W \subset \mathbb{C P}^{m}$ we let $W_{(i)}$ or $W^{(i)}$ denote the corresponding subvariety of $\mathbb{P}_{(i)}^{m}$.

If $P_{i}$ and $P_{j}$ have a common face $F_{i j}$ of dimension $m-1$ then the complexification of the affine span of $F_{i j}$ gives hyperplanes $H_{i j}^{(i)} \subset \mathbb{P}_{(i)}^{m}$ and $H_{i j}^{(j)} \subset \mathbb{P}_{(j)}^{m}$. Moreover, $H_{i j}^{(i)}$ and $H_{i j}^{(j)}$ come with a natural identification $\sigma_{i j}: H_{i j}^{(i)} \cong H_{i j}^{(j)}$. 
We use $\sigma_{i j}$ to glue $\mathbb{P}_{(i)}^{m}$ and $\mathbb{P}_{(j)}^{m}$ together. The resulting variety is isomorphic to the union of 2 hyperplanes in $\mathbb{C P}^{m+1}$.

It is harder to see what happens if we try to perform all these gluings $\sigma_{i j}$ simultaneously.

Let $\amalg_{i} \mathbb{P}_{(i)}^{m}$ denote the disjoint union of all the $\mathbb{P}_{(i)}^{m}$. Each $\sigma_{i j}$ defines a relation that identifies a point $p_{(i)} \in H_{i j}^{(i)} \subset \mathbb{P}_{(i)}^{m}$ with its image $p_{(j)}=$ $\sigma_{i j}\left(p_{(i)}\right) \in H_{i j}^{(j)} \subset \mathbb{P}_{(j)}^{m}$. Let $\Sigma$ denote the equivalence relation generated by all the $\sigma_{i j}$.

It is easy to see (cf. [Kol12, Lem.17]) that there is a projective algebraic variety

$$
\amalg_{i} \mathbb{P}_{(i)}^{m} \longrightarrow\left(\amalg_{i} \mathbb{P}_{(i)}^{m}\right) / \Sigma \longrightarrow \mathbb{C P}^{m}
$$

whose points are exactly the equivalence classes of $\Sigma$.

This gives the correct simple normal crossing variety if $m=1$ but already for $m=2$ we have problems. For instance, consider three 2-cells $P_{i}, P_{j}, P_{k}$ such that $P_{i}$ and $P_{j}$ have a common face $F_{i j}, P_{j}$ and $P_{k}$ have a common face $F_{j k}$ but $P_{i} \cap P_{k}=\emptyset$. The problem is that while $F_{i j}$ and $F_{j k}$ are disjoint, their complexified spans are lines in $\mathbb{C P}^{2}$ hence they intersect at a point $q$. Thus $\sigma_{i j}$ identifies $q_{(i)} \in \mathbb{P}_{(i)}^{2}$ with $q_{(j)} \in \mathbb{P}_{(j)}^{2}$ and $\sigma_{j k}$ identifies $q_{(j)} \in \mathbb{P}_{(j)}^{2}$ with $q_{(k)} \in \mathbb{P}_{(k)}^{2}$ thus the equivalence relation $\Sigma$ identifies $q_{(i)} \in \mathbb{P}_{(i)}^{2}$ with $q_{(k)} \in \mathbb{P}_{(k)}^{2}$. Thus in $\left(\amalg_{i} \mathbb{P}_{(i)}^{m}\right) / \Sigma$ the images of $\mathbb{P}_{(i)}^{2}$ and of $\mathbb{P}_{(k)}^{2}$ are not disjoint.

In order to get the correct simple normal crossing variety, we need to remove these extra intersection points. In higher dimensions we need to remove various linear subspaces as well.

Definition 27 (Essential and parasitic intersections). Let $\mathcal{C}$ be a Voronoi complex on $\mathbb{R}^{m}$ defined by the points $\left\{y_{i}: i \in I\right\}$. We have the linear subspaces $H_{J}$ defined in (24). Assume for simplicity that $J_{1} \neq J_{2}$ implies that $H_{J_{1}} \neq H_{J_{2}}$.

Let $P \subset \mathbb{R}^{m}$ be a Voronoi cell. We say that $H_{J}$ is essential for $P$ if it is the affine span of a face of $P$. Otherwise it is called parasitic for $P$.

Lemma 28. Let $P \subset \mathbb{R}^{m}$ be a simple Voronoi cell.

(1) Every essential subspace $L$ of dimension $\leq m-2$ is contained in a unique smallest parasitic subspace which has dimension $\operatorname{dim} L+1$.

(2) The intersection of two parasitic subspaces is again parasitic.

Proof. There is a point $y_{p} \in P$ and a subset $J \subset I$ such that $H_{i p}$ are spans of faces of $P$ for $i \in J$ and $L=\cap_{i \in J} H_{i p}$. Thus the unique $\operatorname{dim} L+1$ dimensional parasitic subspace containing $L$ is $H_{J}$.

Assume that $L_{1}, L_{2}$ are parasitic. If $L_{1} \cap L_{2}$ is essential then there is a unique smallest parasitic subspace $L^{\prime} \supset L_{1} \cap L_{2}$. Then $L^{\prime} \subset L_{i}$ a contradiction. 
29 (Removing parasitic intersections). Let $\left\{H_{s}: s \in S\right\}$ be a finite set of hyperplanes of $\mathbb{C P}^{m}$. For $Q \subset S$ set $H_{Q}:=\cap_{s \in Q} H_{s}$. Let $\mathcal{P} \subset 2^{S}$ be a subset closed under unions.

Set $\pi_{0}: P^{0} \cong \mathbb{C P}^{m}$. If $\pi_{r}: P^{r} \rightarrow \mathbb{C P}^{m}$ is already defined then let $P^{r+1} \rightarrow P^{r}$ denote the blow-up of the union of birational transforms of all the $H_{Q}$ such that $Q \in \mathcal{P}$ and $\operatorname{dim} H_{Q}=r$. Then $\pi_{r+1}$ is the composite $P^{r+1} \rightarrow P^{r} \rightarrow \mathbb{C P}^{m}$.

Note that we blow up a disjoint union of smooth subvarieties since any intersection of the $r$-dimensional $H_{Q}$ is lower dimensional, hence it was removed by an earlier blow up. Finally set $\Pi: \tilde{P}:=P^{m-2} \rightarrow \mathbb{C P}^{m}$.

Let $\mathcal{C}$ be a pure dimensional subcomplex of a Voronoi complex as in (25). For each cell $P_{i} \in \mathcal{C}$ we use (29) with

$$
\mathcal{P}_{i}:=\left\{\text { parasitic intersections for } P_{i}\right\}
$$

to obtain $\tilde{P}_{(i)}$. Note that if $P_{i}$ and $P_{j}$ have a common codimension 1 face $F_{i j}$ then we perform the same blow-ups on the complexifications $H_{i j}^{(i)} \subset \mathbb{P}_{(i)}^{m}$ and $H_{i j}^{(j)} \subset \mathbb{P}_{(j)}^{m}$. Thus $\sigma_{i j}: H_{i j}^{(i)} \cong H_{i j}^{(j)}$ lifts to the birational transforms

$$
\tilde{\sigma}_{i j}: \tilde{H}_{i j}^{(i)} \cong \tilde{H}_{i j}^{(j)} \text {. }
$$

As before, the $\tilde{\sigma}_{i j}$ define an equivalence relation $\tilde{\Sigma}$ on $\amalg_{i} \tilde{P}_{(i)}$. With these changes, the approach outlined in Paragraph 26 does work and we get the following.

TheOREM 30. [KK11, Prop.28] With the above notation there is a projective, simple normal crossing variety

$$
V(\mathcal{C}):=\left(\amalg_{i} \tilde{P}_{(i)}\right) / \tilde{\Sigma}
$$

with the following properties.

(1) There is a finite morphism $\amalg_{i} \tilde{P}_{(i)} \longrightarrow V(\mathcal{C})$ whose fibers are exactly the equivalence classes of $\tilde{\Sigma}$.

(2) The dual complex $\mathcal{D}(V(\mathcal{C}))$ is naturally identified with the Delaunay triangulation of $\mathcal{C}$.

Comments on the proof. The existence of $V(\mathcal{C})$ is relatively easy either directly as in [KK11, Prop.31] or using the general theory of quotients by finite equivalence relations as in [Kol12].

As we noted in Paragraph 18 the projectivity of such quotients is a rather delicate question since the maps $\tilde{P}_{(i)} \rightarrow \mathbb{C P}^{m}$ are not finite any more.

The main advantage we have here is that each $\tilde{P}_{(i)}$ comes with a specific sequence of blow-ups $\Pi_{i}: \tilde{P}_{(i)} \rightarrow \mathbb{C P}^{m}$ and this enables us to write down explicit, invertible, ample subsheaves $A_{i} \subset \Pi_{i}^{*} \mathcal{O}_{\mathbb{C P} m}(N)$ for some $N \gg 1$ that glue together to give an ample invertible sheaf on $V(T)$. For details see [KK11, Par.32].

The culmination of the results of the last 2 sections is the following. 
Theorem 31. [KK11, Thm.29] Let $T$ be a finite cell complex. Then there is a projective simple normal crossing variety $Z_{T}$ such that

(1) $\mathcal{D}\left(Z_{T}\right)$ is homotopy equivalent to $T$,

(2) $\pi_{1}\left(Z_{T}\right) \cong \pi_{1}(T)$ and

(3) $H^{i}\left(Z_{T}, \mathcal{O}_{Z_{T}}\right) \cong H^{i}(T, \mathbb{C})$ for every $i \geq 0$.

Proof. We have already established (1) in (30), moreover the construction yields a simple normal crossing variety $Z_{T}$ whose strata are all rational varieties. In particular every stratum $W \subset Z_{T}$ is simply connected and $H^{r}\left(W, \mathcal{O}_{W}\right)=0$ for every $r>0$. Thus $(2-3)$ follow from Lemmas 32-33.

The proof of the following lemma is essentially in [GS75, pp.68-72]. More explicit versions can be found in [FM83, pp.26-27] and [Ish85, ABW09].

LEMma 32. Let $X$ be a simple normal crossing variety over $\mathbb{C}$ with irreducible components $\left\{X_{i}: i \in I\right\}$. Let $T=D(X)$ be the dual complex of $X$.

(1) There are natural injections $H^{r}(T, \mathbb{C}) \hookrightarrow H^{r}\left(X, \mathcal{O}_{X}\right)$ for every $r$.

(2) Assume that $H^{r}\left(W, \mathcal{O}_{W}\right)=0$ for every $r>0$ and for every stratum $W \subset X$. Then $H^{r}\left(X, \mathcal{O}_{X}\right)=H^{r}(T, \mathbb{C})$ for every $r$.

The following comparison result is rather straightforward.

Lemma 33. [Cor92, Prop.3.1] Using the notation of (32) assume that every stratum $W \subset X$ is 1 -connected. Then $\pi_{1}(X) \cong \pi_{1}(\mathcal{D}(X))$.

\section{Generic embeddings of simple normal crossing varieties}

The following is a summary of the construction of [Kol11]; see also [Kol13b, Sec.3.4] for an improved version.

34. Let $Z$ be a projective, local complete intersection variety of dimension $n$ and choose any embedding $Z \subset P$ into a smooth projective variety of dimension $N$. (We can take $P=\mathbb{P}^{N}$ for $N \gg 1$.) Let $L$ be a sufficiently ample line bundle on $P$. Let $Z \subset Y_{1} \subset P$ be the complete intersection of $(N-n-1)$ general sections of $L(-Z)$. Set

$$
Y:=B_{(-Z)} Y_{1}:=\operatorname{Proj}_{Y_{1}} \sum_{m=0}^{\infty} \mathcal{O}_{Y_{1}}(m Z)
$$

(Note that this is not the blow-up of $Z$ but the blow-up of its inverse in the class group.)

It is proved in [Kol11] that the birational transform of $Z$ in $Y$ is a Cartier divisor isomorphic to $Z$ and there is a contraction morphism

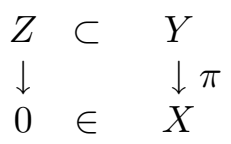

such that $Y \backslash Z \cong X \backslash\{0\}$. If $Y$ is smooth then $\mathcal{D R}(0 \in X)=\mathcal{D}(Z)$ and we are done with Theorem 7 . However, the construction of [Kol11] yields a 
smooth variety $Y$ only if $\operatorname{dim} Z=1$ or $Z$ is smooth. (By (19) this limitation is not unexpected.)

In order to resolve singularities of $Y$ we need a detailed description of them. This is a local question, so we may assume that $Z \subset \mathbb{C}_{\mathrm{x}}^{N}$ is a complete intersection defined by $f_{1}=\cdots=f_{N-n}=0$. Let $Z \subset Y_{1} \subset \mathbb{C}^{N}$ be a general complete intersection defined by equations

$$
h_{i, 1} f_{1}+\cdots+h_{i, N-n} f_{N-n}=0 \quad \text { for } i=1, \ldots, N-n-1 .
$$

Let $H=\left(h_{i j}\right)$ be the $(N-n-1) \times(N-n)$ matrix of the system and $H_{i}$ the submatrix obtained by removing the $i$ th column. By [Kol11] or $[$ Kol13b, Sec.3.2], an open neighborhood of $Z \subset Y$ is defined by the equations

$$
\left(f_{i}=(-1)^{i} \cdot t \cdot \operatorname{det} H_{i}: i=1, \ldots, N-n\right) \subset \mathbb{C}_{\mathbf{x}}^{N} \times \mathbb{C}_{t} .
$$

Assume now that $Z$ has hypersurface singularities. Up-to permuting the $f_{i}$ and passing to a smaller open set, we may assume that $d f_{2}, \ldots, d f_{N-n}$ are linearly independent everywhere along $Z$. Then the singularities of $Y$ all come from the equation

$$
f_{1}=-t \cdot \operatorname{det} H_{1} .
$$

Our aim is to write down local normal forms for $Y$ along $Z$ in the normal crossing case.

On $\mathbb{C}^{N}$ there is a stratification $\mathbb{C}^{N}=R_{0} \supset R_{1} \supset \cdots$ where $R_{i}$ is the set of points where $\operatorname{rank} H_{1} \leq(N-n-1)-i$. Since the $h_{i j}$ are general, $\operatorname{codim}_{W} R_{i}=i^{2}$ and we may assume that every stratum of $Z$ is transversal to each $R_{i} \backslash R_{i+1}$ (cf. Paragraph 37).

Let $S \subset Z$ be any stratum and $p \in S$ a point such that $p \in R_{m} \backslash R_{m+1}$. We can choose local coordinates $\left\{x_{1}, \ldots, x_{d}\right\}$ and $\left\{y_{r s}: 1 \leq r, s \leq m\right\}$ such that, in a neighborhood of $p$,

$$
f_{1}=x_{1} \cdots x_{d} \text { and } \operatorname{det} H_{1}=\operatorname{det}\left(y_{r s}: 1 \leq r, s \leq m\right) .
$$

Note that $m^{2} \leq \operatorname{dim} S=n-d$, thus we can add $n-d-m^{2}$ further coordinates $y_{i j}$ to get a complete local coordinate system on $S$.

Then the $n$ coordinates $\left\{x_{k}, y_{i j}\right\}$ determine a map

$$
\sigma: \mathbb{C}^{N} \times \mathbb{C}_{t} \rightarrow \mathbb{C}^{n} \times \mathbb{C}_{t}
$$

such that $\sigma(Y)$ is defined by the equation

$$
x_{1} \cdots x_{d}=t \cdot \operatorname{det}\left(y_{r s}: 1 \leq r, s \leq m\right) .
$$

Since $d f_{2}, \ldots, d f_{N-n}$ are linearly independent along $Z$, we see that $\left.\sigma\right|_{Y}$ is étale along $Z \subset Y$.

We can summarize these considerations as follows.

Proposition 35. Let $Z$ be a normal crossing variety of dimension $n$. Then there is a normal singularity $(0 \in X)$ of dimension $n+1$ and a proper, birational morphism $\pi: Y \rightarrow X$ such that red $\pi^{-1}(0) \cong Z$ and for every point $p \in \pi^{-1}(0)$ we can choose local (étale or analytic) coordinates called 
$\left\{x_{i}: i \in I_{p}\right\}$ and $\left\{y_{r s}: 1 \leq r, s \leq m_{p}\right\}$ (plus possibly other unnamed coordinates) such that one can write the local equations of $Z \subset Y$ as

$$
\left(\prod_{i \in I_{p}} x_{i}=t=0\right) \subset\left(\prod_{i \in I_{p}} x_{i}=t \cdot \operatorname{det}\left(y_{r s}: 1 \leq r, s \leq m_{p}\right)\right) \subset \mathbb{C}^{n+2} .
$$

36 (Proof of Theorem 7). Let $T$ be a finite cell complex. By (31) there is a projective simple normal crossing variety $Z$ such that $\mathcal{D}(Z)$ is homotopy equivalent to $T, \pi_{1}(Z) \cong \pi_{1}(T)$ and $H^{i}\left(Z, \mathcal{O}_{Z}\right) \cong H^{i}(T, \mathbb{C})$ for every $i \geq 0$.

Then Proposition 35 constructs a singularity $(0 \in X)$ with a partial resolution

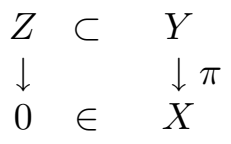

The hardest is to check that we can resolve the singularities of $Y$ without changing the homotopy type of the dual complex of the exceptional divisor. This is done in Section 6.

In order to show $(7.2-3)$ we need further information about the varieties and maps in (36.1).

First, $Y$ has rational singularities. This is easy to read off from their equations. (For the purposes of Theorem 3, we only need the case $\operatorname{dim} Y=3$ when the only singularities we have are ordinary double points with local equation $x_{1} x_{2}=t y_{11}$.)

Second, we can arrange that $Z$ has very negative normal bundle in $Y$. By a general argument this implies that $R^{i} \pi_{*} \mathcal{O}_{Y} \cong H^{i}\left(Z, \mathcal{O}_{Z}\right)$, proving (7.3); see [Kol11, Prop.9] for details.

Finally we need to compare $\pi_{1}(Z)$ with $\pi_{1}(L(0 \in X))$. There is always a surjection

$$
\pi_{1}(L(0 \in X)) \rightarrow \pi_{1}(Z)
$$

but it can have a large kernel. We claim however, that with suitable choices we can arrange that (36.2) is an isomorphism. It is easiest to work not on $Z \subset Y$ but on a resolution $Z^{\prime} \subset Y^{\prime}$.

More generally, let $W$ be a smooth variety, $D=\cup_{i} D_{i} \subset W$ a simple normal crossing divisor and $T \supset D$ a regular neighborhood with boundary $M=\partial T$. There is a natural (up to homotopy) retraction map $T \rightarrow D$ which induces $M \rightarrow D$ hence a surjection $\pi_{1}(M) \rightarrow \pi_{1}(D)$ whose kernel is generated (as a normal subgroup) by the simple loops $\gamma_{i}$ around the $D_{i}$.

In order to understand this kernel, assume first that $D$ is smooth. Then $M \rightarrow D$ is a circle bundle hence there is an exact sequence

$$
\pi_{2}(D) \stackrel{c_{1} \cap}{\longrightarrow} \mathbb{Z} \cong \pi_{1}\left(\mathbb{S}^{1}\right) \rightarrow \pi_{1}(M) \rightarrow \pi_{1}(D) \rightarrow 1
$$

where $c_{1}$ is the Chern class of the normal bundle of $D$ in $X$. Thus if $c_{1} \cap \alpha=1$ for some $\alpha \in \pi_{2}(D)$ then $\pi_{1}(M) \cong \pi_{1}(D)$. In the general case, arguing as above we see that $\pi_{1}(M) \cong \pi_{1}(D)$ if the following holds:

(3) For every $i$ there is a class $\alpha_{i} \in \pi_{2}\left(D_{i}^{0}\right)$ such that $c_{1}\left(N_{D_{i}, X}\right) \cap \alpha_{i}=1$ where $D_{i}^{0}:=D_{i} \backslash\{$ other components of $D\}$. 
Condition (3) is typically very easy to achieve in our constructions. Indeed, we obtain the $D_{i}^{0}$ by starting with $\mathbb{C P}^{m}$, blowing it up many times and then removing a few divisors. Thus we end up with very large $H_{2}\left(D_{i}^{0}, \mathbb{Z}\right)$ and typically the $D_{i}^{0}$ are even simply connected, hence $\pi_{2}\left(D_{i}^{0}\right)=$ $H_{2}\left(D_{i}^{0}, \mathbb{Z}\right)$.

37 (Determinantal varieties). We have used the following basic properties of determinantal varieties. These are quite easy to prove directly; see [Har95, 12.2 and 14.16] for a more general case.

Let $V$ be a smooth, affine variety, and $\mathcal{L} \subset \mathcal{O}_{V}$ a finite dimensional sub vector space without common zeros. Let $H=\left(h_{i j}\right)$ be an $n \times n$ matrix whose entries are general elements in $\mathcal{L}$. For a point $p \in V$ set $m_{p}=$ corank $H(p)$. Then there are local analytic coordinates $\left\{y_{r s}: 1 \leq r, s \leq m_{p}\right\}$ (plus possibly other unnamed coordinates) such that, in a neighborhood of $p$,

$$
\operatorname{det} H=\operatorname{det}\left(y_{r s}: 1 \leq r, s \leq m_{p}\right) \text {. }
$$

In particular, $\operatorname{mult}_{p}(\operatorname{det} H)=\operatorname{corank} H(p)$, for every $m$ the set of points $R_{m} \subset V$ where corank $H(p) \geq m$ is a subvariety of pure codimension $m^{2}$ and Sing $R_{m}=R_{m+1}$.

\section{Resolution of generic embeddings}

In this section we start with the varieties constructed in Proposition 35 and resolve their singularities. Surprisingly, the resolution process described in Paragraphs 39-44 leaves the dual complex unchanged and we get the following.

TheOREM 38. Let $Z$ be a projective simple normal crossing variety of dimension $n$. Then there is a normal singularity $(0 \in X)$ of dimension $(n+1)$ and a resolution $\pi: Y \rightarrow X$ such that $E:=\pi^{-1}(0) \subset Y$ is a simple normal crossing divisor and its dual complex $\mathcal{D}(E)$ is naturally identified with $\mathcal{D}(Z)$. (More precisely, there is a morphism $E \rightarrow Z$ that induces a birational map on every stratum.)

39 (Inductive set-up for resolution). The object we try to resolve is a triple

$$
(Y, E, F):=\left(Y, \sum_{i \in I} E_{i}, \sum_{j \in J} a_{j} F_{j}\right)
$$

where $Y$ is a variety over $\mathbb{C}, E_{i}, F_{j}$ are codimension 1 subvarieties and $a_{j} \in \mathbb{N}$. (The construction (34) produces a triple $(Y, E:=Z, F:=\emptyset)$. The role of the $F_{j}$ is to keep track of the exceptional divisors as we resolve the singularities of $Y$.)

We assume that $E$ is a simple normal crossing variety and for every point $p \in E$ there is a (Euclidean) open neighborhood $p \in Y_{p} \subset Y$, an embedding $\sigma_{p}: Y_{p} \hookrightarrow \mathbb{C}^{\operatorname{dim} Y+1}$ whose image can be described as follows.

There are subsets $I_{p} \subset I$ and $J_{p} \subset J$, a natural number $m_{p} \in \mathbb{N}$ and coordinates in $\mathbb{C}^{\operatorname{dim} Y+1}$ called

$$
\left\{x_{i}: i \in I_{p}\right\},\left\{y_{r s}: 1 \leq r, s \leq m_{p}\right\},\left\{z_{j}: j \in J_{p}\right\} \quad \text { and } \quad t
$$


(plus possibly other unnamed coordinates) such that $\sigma_{p}\left(Y_{p}\right) \subset \mathbb{C}^{\operatorname{dim} Y+1}$ is an open subset of the hypersurface

$$
\prod_{i \in I_{p}} x_{i}=t \cdot \operatorname{det}\left(y_{r s}: 1 \leq r, s \leq m_{p}\right) \cdot \prod_{j \in J_{p}} z_{j}^{a_{j}} .
$$

Furthermore,

$$
\begin{aligned}
& \sigma_{p}\left(E_{i}\right)=\left(t=x_{i}=0\right) \cap \sigma_{p}\left(Y_{p}\right) \quad \text { for } \quad i \in I_{p} \quad \text { and } \\
& \sigma_{p}\left(F_{j}\right)=\left(z_{j}=0\right) \cap \sigma_{p}\left(Y_{p}\right) \quad \text { for } \quad j \in J_{p} .
\end{aligned}
$$

We do not impose any compatibility condition between the local equations on overlapping charts.

We say that $(Y, E, F)$ is resolved at $p$ if $Y$ is smooth at $p$.

The key technical result of this section is the following.

Proposition 40. Let $(Y, E, F)$ be a triple as above. Then there is a resolution of singularities $\pi:\left(Y^{\prime}, E^{\prime}, F^{\prime}\right) \rightarrow(Y, E, F)$ such that

(1) $Y^{\prime}$ is smooth and $E^{\prime}$ is a simple normal crossing divisor,

(2) $E^{\prime}=\pi^{-1}(E)$

(3) every stratum of $E^{\prime}$ is mapped birationally to a stratum of $E$ and

(4) $\pi$ induces an identification $\mathcal{D}\left(E^{\prime}\right)=\mathcal{D}(E)$.

Proof. The resolution will be a composite of explicit blow-ups of smooth subvarieties (except at the last step). We use the local equations to describe the blow-up centers locally. Thus we need to know which locally defined subvarieties make sense globally. For example, choosing a divisor $F_{j_{1}}$ specifies the local divisor $\left(z_{j_{1}}=0\right)$ at every point $p \in F_{j_{1}}$. Similarly, choosing two divisors $E_{i_{1}}, E_{i_{2}}$ gives the local subvarieties $\left(t=x_{i_{1}}=x_{i_{2}}=0\right)$ at every point $p \in E_{i_{1}} \cap E_{i_{2}}$. (Here it is quite important that the divisors $E_{i}$ are themselves smooth. The algorithm does not seem to work if the $E_{i}$ have self-intersections.) Note that by contrast $\left(x_{i_{1}}=x_{i_{2}}=0\right) \subset Y$ defines a local divisor which has no global meaning. Similarly, the vanishing of any of the coordinate functions $y_{r s}$ has no global meaning.

To a point $p \in \operatorname{Sing} E$ we associate the local invariant

$$
\operatorname{Deg}(p):=\left(\operatorname{deg}_{x}(p), \operatorname{deg}_{y}(p), \operatorname{deg}_{z}(p)\right)=\left(\left|I_{p}\right|, m_{p}, \sum_{j \in J_{p}} a_{j}\right) .
$$

It is clear that $\operatorname{deg}_{x}(p)$ and $\operatorname{deg}_{z}(p)$ do not depend on the local coordinates chosen. We see in (42) that $\operatorname{deg}_{y}(p)$ is also well defined if $p \in \operatorname{Sing} E$. The degrees $\operatorname{deg}_{x}(p), \operatorname{deg}_{y}(p), \operatorname{deg}_{z}(p)$ are constructible and upper semi continuous functions on Sing $E$.

Note that $Y$ is smooth at $p$ iff either $\operatorname{Deg}(p)=(1, *, *)$ or $\operatorname{Deg}(p)=$ $(*, 0,0)$. If $\operatorname{deg}_{x}(p)=1$ then we can rewrite the equation (39.2) as

$$
x^{\prime}=t \cdot \prod_{j} z_{j}^{a_{j}} \quad \text { where } \quad x^{\prime}:=x_{1}+t \cdot\left(1-\operatorname{det}\left(y_{r s}\right)\right) \cdot \prod_{j} z_{j}^{a_{j}},
$$

so if $Y$ is smooth then $(Y, E+F)$ has only simple normal crossings along $E$. Thus the resolution constructed in Theorem 38 is a log resolution.

The usual method of Hironaka would start by blowing up the highest multiplicity points. This introduces new and rather complicated exceptional 
divisors and I have not been able to understand how the dual complex changes.

In our case, it turns out to be better to look at a locus where $\operatorname{deg}_{y}(p)$ is maximal but instead of maximizing $\operatorname{deg}_{x}(p) \operatorname{or} \operatorname{deg}_{z}(p)$ we maximize the dimension. Thus we blow up subvarieties along which $Y$ is not equimultiple. Usually this leads to a morass, but our equations separate the variables into distinct groups which makes these blow-ups easy to compute.

One can think of this as mixing the main step of the Hironaka method with the order reduction for monomial ideals (see, for instance, $[\mathbf{K o l 0 7} \mathbf{b}$, Step 3 of 3.111]).

After some preliminary remarks about blow-ups of simple normal crossing varieties the proof of (40) is carried out in a series of steps (42-44).

We start with the locus where $\operatorname{deg}_{y}(p)$ is maximal and by a sequence of blow-ups we eventually achieve that $\operatorname{deg}_{y}(p) \leq 1$ for every singular point $p$. This, however, increases $\operatorname{deg}_{z}$. Then in 3 similar steps we lower the maximum of $\operatorname{deg}_{z}$ until we achieve that $\operatorname{deg}_{z}(p) \leq 1$ for every singular point $p$. Finally we take care of the singular points where $\operatorname{deg}_{y}(p)+\operatorname{deg}_{z}(p) \geq 1$.

41 (Blowing up simple normal crossing varieties). Let $Z$ be a simple normal crossing variety and $W \subset Z$ a subvariety. We say that $W$ has simple normal crossing with $Z$ if for each point $p \in Z$ there is an open neighborhood $Z_{p}$, an embedding $Z_{p} \hookrightarrow \mathbb{C}^{n+1}$ and subsets $I_{p}, J_{p} \subset\{0, \ldots, n\}$ such that

$$
Z_{p}=\left(\prod_{i \in I_{p}} x_{i}=0\right) \text { and } W \cap Z_{p}=\left(x_{j}=0: j \in J_{p}\right) .
$$

This implies that for every stratum $Z_{J} \subset Z$ the intersection $W \cap Z_{J}$ is smooth (even scheme theoretically).

If $W$ has simple normal crossing with $Z$ then the blow-up $B_{W} Z$ is again a simple normal crossing variety. If $W$ is one of the strata of $Z$, then $\mathcal{D}\left(B_{W} Z\right)$ is obtained from $\mathcal{D}(Z)$ by removing the cell corresponding to $W$ and every other cell whose closure contains it. Otherwise $\mathcal{D}\left(B_{W} Z\right)=\mathcal{D}(Z)$. (In the terminology of [Kol13b, Sec.2.4], $B_{W} Z \rightarrow Z$ is a thrifty modification.)

As an example, let $Z=\left(x_{1} x_{2} x_{3}=0\right) \subset \mathbb{C}^{3}$. There are 7 strata and $\mathcal{D}(Z)$ is the 2-simplex whose vertices correspond to the planes $\left(x_{i}=0\right)$.

Let us blow up a point $W=\{p\} \subset Z$ to get $B_{p} Z \subset B_{p} \mathbb{C}^{3}$. Note that the exceptional divisor $E \subset B_{p} \mathbb{C}^{3}$ is not a part of $B_{p} Z$ and $B_{p} Z$ still has 3 irreducible components.

If $p$ is the origin, then the triple intersection is removed and $\mathcal{D}\left(B_{p} Z\right)$ is the boundary of the 2 -simplex.

If $p$ is not the origin, then $B_{p} Z$ still has 7 strata naturally corresponding to the strata of $Z$ and $\mathcal{D}\left(B_{p} Z\right)$ is the 2-simplex.

We will be interested in situations where $Y$ is a hypersurface in $\mathbb{C}^{n+2}$ and $Z \subset Y$ is a Cartier divisor that is a simple normal crossing variety. Let $W \subset Y$ be a smooth, irreducible subvariety, not contained in $Z$ such that

(1) the scheme theoretic intersection $W \cap Z$ has simple normal crossing with $Z$ 
(2) mult $_{Z \cap W} Z=$ mult $_{W} Y$. (Note that this holds if $W \subset \operatorname{Sing} Y$ and mult $_{Z \cap W} Z=2$.)

Choose local coordinates $\left(x_{0}, \ldots, x_{n}, t\right)$ such that $W=\left(x_{0}=\cdots x_{i}=0\right)$ and $Z=(t=0) \subset Y$. Let $f\left(x_{0}, \ldots, x_{n}, t\right)=0$ be the local equation of $Y$.

Blow up $W$ to get $\pi: B_{W} Y \rightarrow Y$. Up to permuting the indices $0, \ldots, i$, the blow-up $B_{W} Y$ is covered by coordinate charts described by the coordinate change

$$
\left(x_{0}, x_{1}, \ldots, x_{i}, x_{i+1}, \ldots, x_{n}, t\right)=\left(x_{0}^{\prime}, x_{1}^{\prime} x_{0}^{\prime}, \ldots, x_{i}^{\prime} x_{0}^{\prime}, x_{i+1}, \ldots, x_{n}, t\right) .
$$

If mult $_{W} Y=d$ then the local equation of $B_{W} Y$ in the above chart becomes

$$
\left(x_{0}^{\prime}\right)^{-d} f\left(x_{0}^{\prime}, x_{1}^{\prime} x_{0}^{\prime}, \ldots, x_{i}^{\prime} x_{0}^{\prime}, x_{i+1}, \ldots, x_{n}, t\right)=0 .
$$

By assumption $(2),\left(x_{0}^{\prime}\right)^{d}$ is also the largest power that divides

$$
f\left(x_{0}^{\prime}, x_{1}^{\prime} x_{0}^{\prime}, \ldots, x_{i}^{\prime} x_{0}^{\prime}, x_{i+1}, \ldots, x_{n}, 0\right),
$$

hence $\pi^{-1}(Z)=B_{W \cap Z} Z$.

Observe finally that the conditions (1-2) can not be fulfilled in any interesting way if $Y$ is smooth. Since we want $Z \cap W$ to be scheme theoretically smooth, if $Y$ is smooth then condition (1) implies that $Z \cap W$ is disjoint from Sing $Z$.

(As an example, let $Y=\mathbb{C}^{3}$ and $Z=(x y z=0)$. Take $W:=(x=y=z)$. Note that $W$ is transversal to every irreducible component of $Z$ but $W \cap Z$ is a non-reduced point. The preimage of $Z$ in $B_{W} Y$ does not have simple normal crossings.)

There are, however, plenty of examples where $Y$ is singular along $Z \cap W$ and these are exactly the singular points that we want to resolve.

42 (Resolving the determinantal part). Let $m$ be the largest size of a determinant occurring at a non-resolved point. Assume that $m \geq 2$ and let $p \in Y$ be a non-resolved point with $m_{p}=m$.

Away from $E \cup F$ the local equation of $Y$ is

$$
\prod_{i \in I_{p}} x_{i}=\operatorname{det}\left(y_{r s}: 1 \leq r, s \leq m\right) .
$$

Thus, the singular set of $Y_{p} \backslash(E \cup F)$ is

$$
\bigcup_{\left(i, i^{\prime}\right)}\left(\operatorname{rank}\left(y_{r s}\right) \leq m-2\right) \cap\left(x_{i}=x_{i^{\prime}}=0\right)
$$

where the union runs through all 2-element subsets $\left\{i, i^{\prime}\right\} \subset I_{p}$. Thus the irreducible components of $\operatorname{Sing} Y \backslash(E \cup F)$ are in natural one-to-one correspondence with the irreducible components of $\operatorname{Sing} E$ and the value of $m=\operatorname{deg}_{y}(p)$ is determined by the multiplicity of any of these irreducible components at $p$.

Pick $i_{1}, i_{2} \in I$ and we work locally with a subvariety

$$
W_{p}^{\prime}\left(i_{1}, i_{2}\right):=\left(\operatorname{rank}\left(y_{r s}\right) \leq m-2\right) \cap\left(x_{i_{1}}=x_{i_{2}}=0\right) .
$$


Note that $W_{p}^{\prime}\left(i_{1}, i_{2}\right)$ is singular if $m>2$ and the subset of its highest multiplicity points is given by $\operatorname{rank}\left(y_{r s}\right)=0$. Therefore the locally defined subvarieties

$$
W_{p}\left(i_{1}, i_{2}\right):=\left(y_{r s}=0: 1 \leq r, s \leq m\right) \cap\left(x_{i_{1}}=x_{i_{2}}=0\right) .
$$

glue together to a well defined global smooth subvariety $W:=W\left(i_{1}, i_{2}\right)$.

$E$ is defined by $(t=0)$ thus $E \cap W$ has the same local equations as $W_{p}\left(i_{1}, i_{2}\right)$. In particular, $E \cap W$ has simple normal crossings with $E$ and $E \cap W$ is not a stratum of $E$; its codimension in the stratum $\left(x_{i_{1}}=x_{i_{2}}=0\right)$ is $m^{2}$.

Furthermore, $E$ has multiplicity 2 along $E \cap W$, hence (41.2) also holds and so

$$
\mathcal{D}\left(B_{E \cap W}\right)=\mathcal{D}(E) .
$$

We blow up $W \subset Y$. We will check that the new triple is again of the form (39). The local degree $\operatorname{Deg}(p)$ is unchanged over $Y \backslash W$. The key assertion is that, over $W$, the maximum value of $\operatorname{Deg}(p)$ (with respect to the lexicographic ordering) decreases. By repeating this procedure for every irreducible components of Sing $E$, we decrease the maximum value of $\operatorname{Deg}(p)$. We can repeat this until we reach $\operatorname{deg}_{y}(p) \leq 1$ for every non-resolved point $p \in Y$.

(Note that this procedure requires an actual ordering of the irreducible components of Sing $E$, which is a non-canonical choice. If a finite groups acts on $Y$, our resolution usually can not be chosen equivariant.)

Now to the local computation of the blow-up. Fix a point $p \in W$ and set $I_{p}^{*}:=I_{p} \backslash\left\{i_{1}, i_{2}\right\}$. We write the local equation of $Y$ as

$$
x_{i_{1}} x_{i_{2}} \cdot L=t \cdot \operatorname{det}\left(y_{r s}\right) \cdot R \quad \text { where } \quad L:=\prod_{i \in I_{p}^{*}} x_{i} \quad \text { and } \quad R:=\prod_{j \in J_{p}} z_{j}^{a_{j}} .
$$

Since $W=\left(x_{i_{1}}=x_{i_{2}}=y_{r s}=0: 1 \leq r, s \leq m\right)$ there are two types of local charts on the blow-up.

(1) There are two charts of the first type. Up to interchanging the subscripts 1,2 , these are given by the coordinate change

$$
\left(x_{i_{1}}, x_{i_{2}}, y_{r s}: 1 \leq r, s \leq m\right)=\left(x_{i_{1}}^{\prime}, x_{i_{2}}^{\prime} x_{i_{1}}^{\prime}, y_{r s}^{\prime} x_{i_{1}}^{\prime}: 1 \leq r, s \leq m\right) .
$$

After setting $z_{w}:=x_{i_{1}}^{\prime}$ the new local equation is

$$
x_{i_{2}}^{\prime} \cdot L=t \cdot \operatorname{det}\left(y_{r s}^{\prime}\right) \cdot\left(z_{w}^{m^{2}-2} \cdot R\right) .
$$

The exceptional divisor is added to the $F$-divisors with coefficient $m^{2}-2$ and the new degree is $\left(\operatorname{deg}_{x}(p)-1, \operatorname{deg}_{y}(p), \operatorname{deg}_{z}(p)+m^{2}-2\right)$.

(2) There are $m^{2}$ charts of the second type. Up to re-indexing the $m^{2}$ pairs $(r, s)$ these are given by the coordinate change

$$
\left(x_{i_{1}}, x_{i_{2}}, y_{r s}: 1 \leq r, s \leq m\right)=\left(x_{i_{1}}^{\prime} y_{m m}^{\prime \prime}, x_{i_{2}}^{\prime} y_{m m}^{\prime \prime}, y_{r s}^{\prime} y_{m m}^{\prime \prime}: 1 \leq r, s \leq m\right)
$$


except when $r=s=m$ where we set $y_{m m}=y_{m m}^{\prime \prime}$. It is convenient to set $y_{m m}^{\prime}=1$ and $z_{w}:=y_{m m}^{\prime \prime}$. Then the new local equation is

$$
x_{i_{1}}^{\prime} x_{i_{2}}^{\prime} \cdot L=t \cdot \operatorname{det}\left(y_{r s}^{\prime}: 1 \leq r, s \leq m\right) \cdot\left(z_{w}^{m^{2}-2} \cdot R\right) .
$$

Note that the $(m, m)$ entry of $\left(y_{r s}^{\prime}\right)$ is 1 . By row and column operations we see that

$$
\operatorname{det}\left(y_{r s}^{\prime}: 1 \leq r, s, \leq m\right)=\operatorname{det}\left(y_{r s}^{\prime}-y_{r m}^{\prime} y_{m s}^{\prime}: 1 \leq r, s, \leq m-1\right) \text {. }
$$

By setting $y_{r s}^{\prime \prime}:=y_{r s}^{\prime}-y_{r m}^{\prime} y_{m s}^{\prime}$ we have new local equations

$$
x_{i_{1}}^{\prime} x_{i_{2}}^{\prime} L=t \cdot \operatorname{det}\left(y_{r s}^{\prime \prime}: 1 \leq r, s, \leq m-1\right) \cdot\left(z_{w}^{m^{2}-2} \cdot R\right)
$$

and the new degree is $\left(\operatorname{deg}_{x}(p), \operatorname{deg}_{y}(p)-1, \operatorname{deg}_{z}(p)+m^{2}-2\right)$.

Outcome. After these blow ups we have a triple $(Y, E, F)$ such that at non-resolved points the local equations are

$$
\prod_{i \in I_{p}} x_{i}=t \cdot y \cdot \prod_{j \in J_{p}} z_{j}^{a_{j}} \quad \text { or } \quad \prod_{i \in I_{p}} x_{i}=t \cdot \prod_{j \in J_{p}} z_{j}^{a_{j}} .
$$

(Note that we can not just declare that $y$ is also a $z$-variable. The $z_{j}$ are local equations of the divisors $F_{j}$ while $(y=0)$ has no global meaning.)

43 (Resolving the monomial part). Following (42.3), the local equations are

$$
\prod_{i \in I_{p}} x_{i}=t \cdot y^{c} \cdot \prod_{j \in J_{p}} z_{j}^{a_{j}} \quad \text { where } c \in\{0,1\} .
$$

We lower the degree of the $z$-monomial in 3 steps.

Step 1. Assume that there is a non-resolved point with $a_{j_{1}} \geq 2$.

The singular set of $F_{j_{1}}$ is then

$$
\bigcup_{\left(i, i^{\prime}\right)}\left(z_{j_{1}}=x_{i}=x_{i^{\prime}}=0\right)
$$

where the union runs through all 2-element subsets $\left\{i, i^{\prime}\right\} \subset I$. Pick an irreducible component of it, call it $W\left(i_{1}, i_{2}, j_{1}\right):=\left(z_{j_{1}}=x_{i_{1}}=x_{i_{2}}=0\right)$.

Set $I_{p}^{*}:=I_{p} \backslash\left\{i_{1}, i_{2}\right\}, J_{p}^{*}:=J_{p} \backslash\left\{j_{1}\right\}$ and write the local equations as

$$
x_{i_{1}} x_{i_{2}} \cdot L=t z_{j}^{a_{j}} \cdot R \quad \text { where } \quad L:=\prod_{i \in I_{p}^{*}} x_{i} \quad \text { and } \quad R:=y^{c} \cdot \prod_{j \in J_{p}^{*}} z_{j}^{a_{j}} .
$$

There are 3 local charts on the blow-up:

(1) $\left(x_{i_{1}}, x_{i_{2}}, z_{j}\right)=\left(x_{i_{1}}^{\prime}, x_{i_{2}}^{\prime} x_{i_{1}}^{\prime}, z_{j}^{\prime} x_{i_{1}}^{\prime}\right)$ and, after setting $z_{w}:=x_{i_{1}}^{\prime}$ the new local equation is

$$
x_{i_{2}}^{\prime} \cdot L=t \cdot z_{w}^{a_{j}-2} z_{j}^{\prime a_{j}} \cdot R .
$$

The new degree is $\left(\operatorname{deg}_{x}(p)-1, \operatorname{deg}_{y}(p), \operatorname{deg}_{z}(p)+a_{j}-2\right)$.

(2) Same as above with the subscripts 1,2 interchanged.

(3) $\left(x_{i_{1}}, x_{i_{2}}, z_{j}\right)=\left(x_{i_{1}}^{\prime} z_{j}^{\prime}, x_{i_{2}}^{\prime} z_{j}^{\prime}, z_{j}^{\prime}\right)$ with new local equation

$$
x_{i_{1}}^{\prime} x_{i_{2}}^{\prime} \cdot L=t \cdot z_{j}^{\prime a_{j}-2} \cdot R .
$$

The new degree is $\left(\operatorname{deg}_{x}(p), \operatorname{deg}_{y}(p), \operatorname{deg}_{z}(p)-2\right)$. 
Step 2. Assume that there is a non-resolved point with $a_{j_{1}}=a_{j_{2}}=1$.

The singular set of $F_{j_{1}} \cap F_{j_{2}}$ is then

$$
\bigcup_{\left(i, i^{\prime}\right)}\left(z_{j_{1}}=z_{j_{2}}=x_{i}=x_{i^{\prime}}=0\right) .
$$

where the union runs through all 2-element subsets $\left\{i, i^{\prime}\right\} \subset I$. Pick an irreducible component of it, call it $W\left(i_{1}, i_{2}, j_{1}, j_{2}\right):=\left(z_{j_{1}}=z_{j_{2}}=x_{i_{1}}=\right.$ $\left.x_{i_{2}}=0\right)$.

Set $I_{p}^{*}:=I_{p} \backslash\left\{i_{1}, i_{2}\right\}, J_{p}^{*}:=J_{p} \backslash\left\{j_{1}, j_{2}\right\}$ and we write the local equations as

$$
x_{i_{1}} x_{i_{2}} \cdot L=t z_{j_{1}} z_{j_{2}} \cdot R \quad \text { where } \quad L:=\prod_{i \in I_{p}^{*}} x_{i} \quad \text { and } \quad R:=y^{c} \cdot \prod_{j \in J_{p}^{*}} z_{j}^{a_{j}} .
$$

There are two types of local charts on the blow-up.

(1) In the chart $\left(x_{i_{1}}, x_{i_{2}}, z_{j_{1}}, z_{j_{2}}\right)=\left(x_{i_{1}}^{\prime}, x_{i_{2}}^{\prime} x_{i_{1}}^{\prime}, z_{j_{1}}^{\prime} x_{i_{1}}^{\prime}, z_{j_{2}}^{\prime} x_{i_{1}}^{\prime}\right)$ the new local equation is

$$
x_{i_{2}}^{\prime} \cdot L=t \cdot z_{j_{1}}^{\prime} z_{j_{2}}^{\prime} \cdot R .
$$

and the new degree is $\left(\operatorname{deg}_{x}(p)-1, \operatorname{deg}_{y}(p), \operatorname{deg}_{z}(p)\right)$. A similar chart is obtained by interchanging the subscripts $i_{1}, i_{2}$.

(2) In the chart $\left(x_{i_{1}}, x_{i_{2}}, z_{j_{1}}, z_{j_{2}}\right)=\left(x_{i_{1}}^{\prime} z_{j_{1}}^{\prime}, x_{i_{2}}^{\prime} z_{j_{1}}^{\prime}, z_{j_{1}}^{\prime}, z_{j_{2}}^{\prime} z_{j_{1}}^{\prime}\right)$. the new local equation is

$$
x_{i_{1}}^{\prime} x_{i_{2}}^{\prime} \cdot L=t \cdot z_{j_{2}}^{\prime} \cdot R .
$$

The new degree is $\left(\operatorname{deg}_{x}(p), \operatorname{deg}_{y}(p), \operatorname{deg}_{z}(p)-1\right)$.

A similar chart is obtained by interchanging the subscripts $j_{1}, j_{2}$.

By repeated application of these two steps we are reduced to the case where $\operatorname{deg}_{z}(p) \leq 1$ at all non-resolved points.

Step 3. Assume that there is a non-resolved point with $\operatorname{deg}_{y}(p)=$ $\operatorname{deg}_{z}(p)=1$.

The singular set of $Y$ is

$$
\bigcup_{\left(i, i^{\prime}\right)}\left(y=z=x_{i}=x_{i^{\prime}}=0\right) .
$$

Pick an irreducible component of it, call it $W\left(i_{1}, i_{2}\right):=\left(y=z=x_{i_{1}}=\right.$ $\left.x_{i_{2}}=0\right)$. The blow up computation is the same as in Step 2.

As before we see that at each step the conditions (41.1-2) hold, hence $\mathcal{D}(E)$ is unchanged.

Outcome. After these blow-ups we have a triple $(Y, E, F)$ such that at non-resolved points the local equations are

$$
\prod_{i \in I_{p}} x_{i}=t \cdot y, \quad \prod_{i \in I_{p}} x_{i}=t \cdot z_{1} \quad \text { or } \quad \prod_{i \in I_{p}} x_{i}=t .
$$

As before, the $y$ and $z$ variables have different meaning, but we can rename $z_{1}$ as $y$. Thus we have only one non-resolved local form left: $\prod x_{i}=t y$. 
44 (Resolving the multiplicity 2 part). Here we have a local equation $x_{i_{1}} \cdots x_{i_{d}}=t y$ where $d \geq 2$. We would like to blow up $\left(x_{i_{1}}=y=0\right)$, but, as we noted, this subvariety is not globally defined. However, a rare occurrence helps us out. Usually the blow-up of a smooth subvariety determines its center uniquely. However, this is not the case for codimension 1 centers. Thus we could get a globally well defined blow-up even from centers that are not globally well defined.

Note that the inverse of $\left(x_{i_{1}}=y=0\right)$ in the local Picard group of $Y$ is $E_{i_{1}}=\left(x_{i_{1}}=t=0\right)$, which is globally defined. Thus

$$
\operatorname{Proj}_{Y} \sum_{m \geq 0} \mathcal{O}_{Y}\left(m E_{i_{1}}\right)
$$

is well defined, and locally it is isomorphic to the blow-up $B_{\left(x_{i_{1}}=y=0\right)} Y$. (A priori, we would need to take the normalization of $B_{\left(x_{i_{1}}=y=0\right)} Y$, but it is actually normal.) Thus we have 2 local charts.

(1) $\left(x_{i_{1}}, y\right)=\left(x_{i_{1}}^{\prime}, y^{\prime} x_{i_{1}}^{\prime}\right)$ and the new local equation is $\left(x_{i_{2}} \cdots x_{i_{d}}=\right.$ $\left.t y^{\prime}\right)$. The new local degree is $(d-1,1,0)$.

(2) $\left(x_{i_{1}}, y\right)=\left(x_{i_{1}}^{\prime} y^{\prime}, y^{\prime}\right)$ and the new local equation is $\left(x_{i_{1}}^{\prime} \cdot x_{i_{2}} \cdots x_{i_{d}}=\right.$ $t)$. The new local degree is $(d, 0,0)$.

Outcome. After all these blow-ups we have a triple $\left(Y, \sum_{i \in I} E_{i}\right.$, $\left.\sum_{j \in J} a_{j} F_{j}\right)$ where $\sum_{i \in I} E_{i}$ is a simple normal crossing divisor and $Y$ is smooth along $\sum_{i \in I} E_{i}$.

This completes the proof of Proposition 40.

45 (Proof of Theorem 8). Assume that $T$ is $\mathbb{Q}$-acyclic. Then, by (31) there is a simple normal crossing variety $Z_{T}$ such that $H^{i}\left(Z_{T}, \mathcal{O}_{Z_{T}}\right)=0$ for $i>0$. Then [Kol11, Prop.9] shows that, for $L$ sufficiently ample, the singularity $\left(0 \in X_{T}\right)$ constructed in (34) and (35) is rational. By (40) we conclude that $\mathcal{D} \mathcal{R}\left(0 \in X_{T}\right) \cong \mathcal{D}\left(Z_{T}\right)$ is homotopy equivalent to $T$.

\section{Cohen-Macaulay singularities}

Definition 46. Cohen-Macaulay singularities form the largest class where Serre duality holds. That is, if $X$ is a projective variety of pure dimension $n$ then $X$ has Cohen-Macaulay singularities iff $H^{i}(X, L)$ is dual to $H^{n-i}\left(X, \omega_{X} \otimes L^{-1}\right)$ for every line bundle $L$. A pleasant property is that if $D \subset X$ is Cartier divisor in a scheme then $D$ is Cohen-Macaulay iff $X$ is Cohen-Macaulay in a neighborhood of D. See [Har77, pp.184-186] or [KM98b, Sec.5.5] for details.

For local questions it is more convenient to use a characterization using local cohomology due to [Gro67, Sec.3.3]: $X$ is Cohen-Macaulay iff $H_{x}^{i}\left(X, \mathcal{O}_{X}\right)=0$ for every $x \in X$ and $i<\operatorname{dim} X$.

Every normal surface is Cohen-Macaulay, so the topology of the links of Cohen-Macaulay singularities starts to become interesting when $\operatorname{dim} X \geq 3$. 
Definition 47. Recall that a group $G$ is called perfect if it has no nontrivial abelian quotients. Equivalently, if $G=[G, G]$ or if $H_{1}(G, \mathbb{Z})=0$.

We say that $G$ is $\mathbb{Q}$-perfect if every abelian quotient is torsion. Equivalently, if $H_{1}(G, \mathbb{Q})=0$.

The following theorem describes the fundamental group of the link of Cohen-Macaulay singularities. Note, however, that the most natural part is the equivalence (48.1) $\Leftrightarrow$ (48.5), relating the fundamental group of the link to the vanishing of $R^{1} f_{*} \mathcal{O}_{Y}$ for a resolution $f: Y \rightarrow X$.

THEOREM 48. For a finitely presented group $G$ the following are equivalent.

(1) $G$ is $\mathbb{Q}$-perfect (47).

(2) $G$ is the fundamental group of the link of an isolated CohenMacaulay singularity of dimension $=3$.

(3) $G$ is the fundamental group of the link of an isolated CohenMacaulay singularity of dimension $\geq 3$.

(4) $G$ is the fundamental group of the link of a Cohen-Macaulay singularity whose singular set has codimension $\geq 3$.

(5) $G$ is the fundamental group of the link of a 1-rational singularity (52).

Proof. It is clear that $(2) \Rightarrow(3) \Rightarrow(4)$ and (49) shows that $(4) \Rightarrow(5)$.

The implication $(5) \Rightarrow(1)$ is proved in (51).

Let us prove $(1) \Rightarrow(2)$. By (31) there is a simple normal crossing variety $Z$ such that $\pi_{1}(Z) \cong G$. By a singular version of the Lefschetz hyperplane theorem (see, for instance, [GM88, Sec.II.1.2]), by taking general hyperplane sections we obtain a simple normal crossing surface $S$ such that $\pi_{1}(S) \cong G$. Thus $H^{1}(S, \mathbb{Q})=0$ and by Hodge theory this implies that $H^{1}\left(S, \mathcal{O}_{S}\right)=0$.

By (35) there is a 3-dimensional isolated singularity $(x \in X)$ with a partial resolution $f: Y \rightarrow X$ whose exceptional divisor is $E \cong S$ and $R^{1} f_{*} \mathcal{O}_{Y} \cong H^{1}\left(E, \mathcal{O}_{E}\right)=0$. In this case the singularities of $Y$ are the simplest possible: we have only ordinary nodes with equation $\left(x_{1} x_{2}=t y_{11}\right)$. These are resolved in 1 step by blowing up $\left(x_{1}=t=0\right)$ and they have no effect on our computations.

Thus $X$ is Cohen-Macaulay by (50).

Lemma 49. Let $X$ be a normal variety with Cohen-Macaulay singularities ( $S_{3}$ would be sufficient) and $f: Y \rightarrow X$ a resolution of singularities. Then $\operatorname{Supp} R^{1} f_{*} \mathcal{O}_{Y}$ has pure codimension 2. Thus if $\operatorname{Sing} X$ has codimension $\geq 3$ then $R^{1} f_{*} \mathcal{O}_{Y}=0$.

Proof. By localizing at a generic point of $\operatorname{Supp} R^{1} f_{*} \mathcal{O}_{Y}$ (or by taking a generic hyperplane section) we may assume that $\operatorname{Supp} R^{1} f_{*} \mathcal{O}_{Y}=\{x\}$ is a closed point. Set $E:=f^{-1}(x)$. There is a Leray spectral sequence

$$
H_{x}^{i}\left(X, R^{j} f_{*} \mathcal{O}_{X}\right) \Rightarrow H_{E}^{i+j}\left(Y, \mathcal{O}_{Y}\right)
$$


By a straightforward duality (see, e.g. [Kol13b, 10.44]) $H_{E}^{r}\left(Y, \mathcal{O}_{Y}\right)$ is dual to the stalk of $R^{n-r} f_{*} \omega_{Y}$ which is zero for $r<n$ by [GR70]. Thus (49.1) gives an exact sequence

$$
H_{x}^{1}\left(X, \mathcal{O}_{X}\right) \rightarrow H_{E}^{1}\left(Y, \mathcal{O}_{Y}\right) \rightarrow H_{x}^{0}\left(X, R^{1} f_{*} \mathcal{O}_{X}\right) \rightarrow H_{x}^{2}\left(X, \mathcal{O}_{X}\right) .
$$

If $X$ is Cohen-Macaulay and $\operatorname{dim} X \geq 3$ then $H_{x}^{1}\left(X, \mathcal{O}_{X}\right)=H_{x}^{2}\left(X, \mathcal{O}_{X}\right)=$ 0 , thus

$$
\left(R^{1} f_{*} \mathcal{O}_{X}\right)_{x} \cong H_{x}^{0}\left(X, R^{1} f_{*} \mathcal{O}_{X}\right) \cong H_{E}^{1}\left(Y, \mathcal{O}_{Y}\right)=0
$$

For isolated singularities, one has the following converse

LEMma 50. Let $(x \in X)$ be a normal, isolated singularity with a resolution $f: Y \rightarrow X$. Then $X$ is Cohen-Macaulay iff $R^{i} f_{*} \mathcal{O}_{Y}=0$ for $0<i<n-1$.

Proof. The spectral sequence (49.1) implies that we have isomorphisms

$$
R^{i} f_{*} \mathcal{O}_{Y} \cong H_{x}^{i}\left(X, \mathcal{O}_{X}\right) \text { for } 0<i<n-1
$$

and $H_{x}^{1}\left(X, \mathcal{O}_{X}\right)=0$ since $X$ is normal.

Lemma 51. Let $X$ be a normal variety with 1-rational singularities (52) and $x \in X$ a point with link $L:=L(x \in X)$. Then $H^{1}(L, \mathbb{Q})=0$.

Proof. Let $f: Y \rightarrow X$ be a resolution such that $E:=f^{-1}(x)$ is a simple normal crossing divisor. By $\left[\right.$ Ste83, 2.14] the natural maps $R^{i} f_{*} \mathcal{O}_{Y} \rightarrow$ $H^{i}\left(E, \mathcal{O}_{E}\right)$ are surjective, thus $H^{1}\left(E, \mathcal{O}_{E}\right)=0$ hence $H^{1}(E, \mathbb{Q})=0$ by Hodge theory.

Next we prove that $H^{1}(E, \mathbb{Q})=H^{1}(L, \mathbb{Q})$. Let $x \in N_{X} \subset X$ be a neighborhood of $x$ such that $\partial N_{X}=L$ and $N_{Y}:=f^{-1}\left(N_{X}\right)$ the corresponding neighborhood of $E$ with boundary $\partial N_{Y}:=L_{Y}$. Since $L_{Y} \rightarrow L$ has connected fibers, $H^{1}(L, \mathbb{Q}) \hookrightarrow H^{1}\left(L_{Y}, \mathbb{Q}\right)$ thus it is enough to prove that $H^{1}\left(L_{Y}, \mathbb{Q}\right)=0$. The exact cohomology sequence of the pair $\left(N_{Y}, L_{Y}\right)$ gives

$0=H^{1}(E, \mathbb{Q})=H^{1}\left(N_{Y}, \mathbb{Q}\right) \rightarrow H^{1}\left(L_{Y}, \mathbb{Q}\right) \rightarrow H^{2}\left(N_{Y}, L_{Y}, \mathbb{Q}\right) \stackrel{\alpha}{\rightarrow} H^{2}\left(N_{Y}, \mathbb{Q}\right)$

By Poincaré duality $H^{2}\left(N_{Y}, L_{Y}, \mathbb{Q}\right) \cong H_{2 n-2}\left(N_{Y}, \mathbb{Q}\right)$. Since $N_{Y}$ retracts to $E$ we see that $H_{2 n-2}\left(N_{Y}, \mathbb{Q}\right)$ is freely generated by the classes of exceptional divisors $E=\cup_{i} E_{i}$. The map $\alpha$ sends $\sum m_{i}\left[E_{i}\right]$ to $c_{1}\left(\mathcal{O}_{N_{Y}}\left(\sum m_{i} E_{i}\right)\right)$ and we need to show that the latter are nonzero. This follows from the Hodge index theorem.

\section{Rational singularities}

Definition 52. A quasi projective variety $X$ has rational singularities if for one (equivalently every) resolution of singularities $p: Y \rightarrow X$ and for every algebraic (or holomorphic) vector bundle $F$ on $X$, the natural maps $H^{i}(X, F) \rightarrow H^{i}\left(Y, p^{*} F\right)$ are isomorphisms. Thus, for purposes of computing cohomology of vector bundles, $X$ behaves like a smooth variety. Rational implies Cohen-Macaulay. See [KM98b, Sec.5.1] for details. 
A more frequently used equivalent definition is the following. $X$ has rational singularities iff the higher direct images $R^{i} f_{*} \mathcal{O}_{Y}$ are zero for $i>0$ for one (equivalently every) resolution of singularities $p: Y \rightarrow X$.

We say that $X$ has 1-rational singularities if $R^{1} f_{*} \mathcal{O}_{Y}=0$ for one (equivalently every) resolution of singularities $p: Y \rightarrow X$.

53 (Proof of Theorem 8). Let $p: Y \rightarrow X$ be a resolution of singularities such that $E_{x}:=p^{-1}(x)$ is a simple normal crossing divisor. As we noted in the proof of $(51), R^{i} f_{*} \mathcal{O}_{Y} \rightarrow H^{i}\left(E, \mathcal{O}_{E}\right)$ is surjective, thus $H^{i}\left(E, \mathcal{O}_{E}\right)=0$ hence $H^{i}(\mathcal{D} \mathcal{R}(x \in X), \mathbb{Q})=0$ by $(32)$. Thus $\mathcal{D} \mathcal{R}(x \in X)$ is $\mathbb{Q}$-acyclic.

Conversely, if $T$ is $\mathbb{Q}$-acyclic then Theorem 7 constructs a singularity which is rational by $(7.3)$.

Let $L$ be the link of a rational singularity $(x \in X)$. Since $X$ is CohenMacaulay, we know that $\pi_{1}(L)$ is $\mathbb{Q}$-perfect (48). It is not known what else can one say about fundamental groups of links of rational singularities, but the fundamental group of the dual complex can be completely described.

Definition 54. A group $G$ is called superperfect if $H_{1}(G, \mathbb{Z})=$ $H_{2}(G, \mathbb{Z})=0$; see $\left[\right.$ Ber02]. We say that $G$ is $\mathbb{Q}$-superperfect if $H_{1}(G, \mathbb{Q})=$ $H_{2}(G, \mathbb{Q})=0$. Note that every finite group is $\mathbb{Q}$-superperfect. Other examples are the infinite dihedral group or $\operatorname{SL}(2, \mathbb{Z})$.

Corollary 55. [KK11, Thm.42] Let $(x \in X)$ be a rational singularity. Then $\pi_{1}(\mathcal{D} \mathcal{R}(X))$ is $\mathbb{Q}$-superperfect. Conversely, for every finitely presented, $\mathbb{Q}$-superperfect group $G$ there is a 6 -dimensional rational singularity $(x \in X)$ such that

$$
\pi_{1}(\mathcal{D} \mathcal{R}(X))=\pi_{1}(\mathcal{R}(X))=\pi_{1}(L(x \in X)) \cong G .
$$

Proof. By a slight variant of the results of [Ker69, KM63], for every finitely presented, $\mathbb{Q}$-superperfect group $G$ there is a $\mathbb{Q}$-acyclic, 5dimensional manifold (with boundary) $M$ whose fundamental group is isomorphic to $G$. Using this $M$ in (8) we get a rational singularity $(x \in X)$ as desired.

Note that just applying the general construction would give 11 dimensional examples. See $\left[\mathbf{K K 1 1}\right.$, Sec.7] on how to lower the dimension to $6 .^{3}$

\section{Questions and problems}

\section{Questions about fundamental groups.}

In principle, for any finitely presented group $G$ one can follow the proof of $\left[\right.$ KK11] and construct links $L$ such that $\pi_{1}(L) \cong G$. However, in almost all cases, the general methods lead to very complicated examples. It would be useful to start with some interesting groups and obtain examples that are understandable. For example, Higman's group

$$
H=\left\langle x_{i}: x_{i}\left[x_{i}, x_{i+1}\right], i \in \mathbb{Z} / 4 \mathbb{Z}\right\rangle
$$

is perfect, infinite and contains no proper finite index subgroups [Hig51].

\footnotetext{
${ }^{3} \mathrm{~A}$ different construction giving 4 and 5 dimensional examples is in [Kol13a].
} 
Problem 56. Find an explicit link whose fundamental group is Higman's group. (It would be especially interesting to find examples that occur "naturally" in algebraic geometry.)

Note that our results give links with a given fundamental group but, as far as we can tell, these groups get killed in the larger quasi-projective varieties. (In particular, we do not answer the question [Ser77, p.19] whether Higman's group can be the fundamental group of a smooth variety.) This leads to the following.

QUESTION 57. Let $G$ be a finitely presented group. Is there a quasiprojective variety $X$ with an isolated singularity $(x \in X)$ such that $\pi_{1}(L(x \in$ $X)) \cong G$ and the natural map $\pi_{1}(L(x \in X)) \rightarrow \pi_{1}(X \backslash\{x\})$ is an injection?

As Kapovich pointed out, it is not known if every finitely presented group occurs as a subgroup of the fundamental group of a smooth projective or quasi-projective variety.

We saw in $(55)$ that $\mathbb{Q}$-superperfect groups are exactly those that occur as $\pi_{1}(\mathcal{D} \mathcal{R}(X))$ for rational singularities. Moreover, every $\mathbb{Q}$-superperfect group can be the fundamental group of a link of a rational singularity. However, there are rational singularities such that the fundamental group of their link is not $\mathbb{Q}$-superperfect. As an example, let $S$ be a fake projective quadric whose universal cover is the 2-disc $\mathbb{D} \times \mathbb{D}$ (cf. [Bea96, Ex.X.13.4]). Let $C(S)$ be a cone over $S$ with link $L(S)$. Then

$$
H^{2}(L(S), \mathbb{Q}) \cong H^{2}(S, \mathbb{Q}) / \mathbb{Q} \cong \mathbb{Q}
$$

and the universal cover of $L$ is an $\mathbb{R}$-bundle over $\mathbb{D} \times \mathbb{D}$ hence contractible. Thus

$$
H^{2}\left(\pi_{1}(L(S)), \mathbb{Q}\right) \cong H^{2}(L(S), \mathbb{Q}) \cong \mathbb{Q},
$$

so $\pi_{1}(L(S))$ is not $\mathbb{Q}$-superperfect. This leads us to the following, possibly very hard, question.

Problem 58. Characterize the fundamental groups of links of rational singularities.

In this context it is worthwhile to mention the following.

Conjecture 59 (Carlson-Toledo). The fundamental group of a smooth projective variety is not $\mathbb{Q}$-superperfect (unless it is finite).

More generally, the original conjecture of Carlson and Toledo asserts that the image

$$
\operatorname{im}\left[H^{2}\left(\pi_{1}(X), \mathbb{Q}\right) \rightarrow H^{2}(X, \mathbb{Q})\right]
$$

is nonzero and contains a (possibly degenerate) Kähler class, see $[\mathbf{K o l 9 5}$, 18.16]. For a partial solution see [Rez02].

Our examples show that for every finitely presented group $G$ there is a reducible simple normal crossing surface $S$ such that $\pi_{1}(S) \cong G$. By [Sim10], for every finitely presented group $G$ there is a (very singular) irreducible 
variety $Z$ such that $\pi_{1}(Z) \cong G$. It is natural to hope to combine these results. [Kap12] proves that for every finitely presented group $G$ there is an irreducible surface $S$ with normal crossing and Whitney umbrella singularities (also called pinch points, given locally as $x^{2}=y^{2} z$ ) such that $\pi_{1}(S) \cong G$.

Problem 60. [Sim10] What can one say about the fundamental groups of irreducible surfaces with normal crossing singularities?

Although closely related, the next question should have a quite different answer.

Problem 61. What can one say about the fundamental groups of normal, projective varieties or surfaces? Are these two classes of groups the same?

Many of the known restrictions on fundamental groups of smooth varieties also apply to normal varieties. For instance, the theory of Albanese varieties implies that the rank of $H_{2}(X, \mathbb{Q})$ is even for normal, projective varieties $X$. Another example is the following. By [Siu87] any surjection $\pi_{1}(X) \rightarrow \pi_{1}(C)$ to the fundamental group of a curve $C$ of genus $\geq 2$ factors as

$$
\pi_{1}(X) \stackrel{g_{*}}{\rightarrow} \pi_{1}\left(C^{\prime}\right) \rightarrow \pi_{1}(C)
$$

where $g: X \rightarrow C^{\prime}$ is a morphism. (In general there is no morphism $C^{\prime} \rightarrow C$.)

We claim that this also holds for normal varieties $Y$. Indeed, let $\pi: Y^{\prime} \rightarrow$ $Y$ be a resolution of singularities. Any surjection $\pi_{1}(Y) \rightarrow \pi_{1}(C)$ induces $\pi_{1}\left(Y^{\prime}\right) \rightarrow \pi_{1}(C)$, hence we get a morphism $g^{\prime}: Y^{\prime} \rightarrow C^{\prime}$. Let $B \subset Y^{\prime}$ be an irreducible curve that is contacted by $\pi$. Then $\pi_{1}(B) \rightarrow \pi_{1}(Y)$ is trivial and so is $\pi_{1}(B) \rightarrow \pi_{1}(C)$. If $\left.g^{\prime}\right|_{B}: B \rightarrow C^{\prime}$ is not constant then the induced map $\pi_{1}(B) \rightarrow \pi_{1}\left(C^{\prime}\right)$ has finite index image. This is impossible since the composite $\pi_{1}(B) \rightarrow \pi_{1}\left(C^{\prime}\right) \rightarrow \pi_{1}(C)$ is trivial. Thus $g^{\prime}$ descends to $g: Y \rightarrow C^{\prime}$.

For further such results see [Gro89, GL91, Cat91, Cat96].

Algebraically one can think of the link as the punctured spectrum of the Henselisation (or completion) of the local ring of $x \in X$. Although one can not choose a base point, it should be possible to define an algebraic fundamental group. All the examples in Theorem 3 can be realized on varieties defined over $\mathbb{Q}$. Thus they should have an algebraic fundamental group $\pi_{1}^{\text {alg }}\left(L\left(0 \in X_{\mathbb{Q}}\right)\right)$ which is an extension of the profinite completion of $\pi_{1}(L(0 \in X))$ and of the Galois group $\operatorname{Gal}(\overline{\mathbb{Q}} / \mathbb{Q})$.

Problem 62. Define and describe the possible groups $\pi_{1}^{\text {alg }}\left(L\left(0 \in X_{\mathbb{Q}}\right)\right)$.

\section{Questions about the topology of links.}

We saw that the fundamental groups of links can be quite different from fundamental groups of quasi-projective varieties. However, our results say very little about the cohomology or other topological properties of links. It 
turns out that links have numerous restrictive topological properties. I thank J. Shaneson and L. Maxim for bringing many of these to my attention.

63 (Which manifolds can be links?). Let $M$ be a differentiable manifold that is diffeomeorphic to the link $L$ of an isolated complex singularity of dimension $n$. Then $M$ satisfies the following.

63.1. $\operatorname{dim}_{\mathbb{R}} M=2 n-1$ is odd and $M$ is orientable. Resolution of singularities shows that $M$ is cobordant to 0 .

63.2. The decomposition $\left.T_{X}\right|_{L} \cong T_{L}+N_{L, X}$ shows that $T_{M}$ is stably complex. In particular, its odd integral Stiefel-Whitney classes are zero [Mas61]. (More generally, this holds for orientable real hypersurfaces in complex manifolds.)

63.3. The cohomology groups $H^{i}(L, \mathbb{Q})$ carry a natural mixed Hodge structure; see [PS08, Sec.6.3] for a detailed treatment and references. Using these, $[\mathbf{D H 8 8}]$ proves that the cup product $H^{i}(L, \mathbb{Q}) \times H^{i}(L, \mathbb{Q}) \rightarrow$ $H^{i+j}(L, \mathbb{Q})$ is zero if $i, j<n$ and $i+j \geq n$. In particular, the torus $\mathbb{T}^{2 n-1}$ can not be a link. If $X$ is a smooth projective variety then $X \times \mathbb{S}^{1}$ can not be a link. Further results along this direction are in [PP08].

63.4. By [CS91, p.548], the components of the Todd-Hirzebruch L-genus of $M$ vanish above the middle dimension. More generally, the purity of the Chern classes and weight considerations as in (63.3) show that the $c_{i}\left(\left.T_{X}\right|_{L}\right)$ are torsion above the middle dimension. Thus all Pontryagin classes of $L$ are torsion above the middle dimension. See also [CMS08a, CMS08b] for further results on the topology of singular algebraic varieties which give restrictions on links as special cases.

There is no reason to believe that this list is complete and it would be useful to construct many different links to get some idea of what other restrictions may hold.

Let $(0 \in X) \subset\left(0 \in \mathbb{C}^{N}\right)$ be an isolated singularity of dimension $n$ and $L=X \cap \mathbb{S}^{2 N-1}(\epsilon)$ its link. If $X_{0}:=X$ is smoothable in a family $\left\{X_{t} \subset \mathbb{C}^{N}\right\}$ then $L$ bounds a Stein manifold $U_{t}:=X_{t} \cap \mathbb{B}^{2 N}(\epsilon)$ and $U_{t}$ is homotopic to an $n$-dimensional compact simplicial complex. This imposes strong restrictions on the topology of smoothable links; some of these were used in [PP08]. Interestingly, these restrictions use the integral structure of the cohomology groups. This leads to the following intriguing possibility.

Question 64 . Let $L$ be a link of dimension $2 n-1$. Does $L$ bound a $\mathbb{Q}$-homology manifold $U$ (of dimension $2 n$ ) that is $\mathbb{Q}$-homotopic to an $n$ dimensional, finite simplicial complex?

There is very little evidence to support the above speculation but it is consistent with known restrictions on the topology of links and it would explain many of them. On the other hand, I was unable to find such $U$ even in some simple cases. For instance, if $(0 \in X)$ is a cone over an Abelian 
variety (or a product of curves of genus $\geq 2$ ) of dimension $\geq 2$ then algebraic deformations of $X$ do not produce such a $U$.

Restricting to the cohomology rings, here are two simple questions.

Question 65 (Cohomology of links). Is the sequence of Betti numbers of a complex link arbitrary? Can one describe the possible algebras $H^{*}(L, \mathbb{Q})$ ?

Question 66 (Cohomology of links of weighted cones). We saw in (11) that the first Betti number of the link of a weighted cone (of dimension $>1$ ) is even. One can ask if this is the only restriction on the Betti numbers of a complex link of a weighted cone.

Philosophically, one of the main results on the topology of smooth projective varieties, proved in [DGMS75, Sul77], says that for simply connected varieties the integral cohomology ring and the Pontryagin classes determine the differentiable structure up to finite ambiguity. It is natural to ask what happens for links.

Question 67. To what extent is the diffeomorphism type of a simply connected link $L$ determined by the cohomology ring $H^{*}(L, \mathbb{Z})$ plus some characteristic classes?

A positive answer to (67) would imply that general links are indeed very similar to weighted homogeneous links and to projective varieties.

Questions about $\mathcal{D} \mathcal{R}(0 \in X)$.

The preprint version contained several questions about dual complexes of dlt pairs; these are corrected and solved in [dFKX12].

Embeddings of simple normal crossing varieties.

In many contexts it has been a difficulty that not every variety with simple normal crossing singularities can be realized as a hypersurface in a smooth variety. See for instance [Fuj09, BM11, BP11, Kol13b] for such examples and for various partial solutions.

As we discussed in (19), recent examples of [Fuj12a, Fuj12b] show that the answer to the following may be quite complicated.

Question 68. Which proper, complex, simple normal crossing spaces can be realized as hypersurfaces in a complex manifold?

QUESTION 69. Which projective simple normal crossing varieties can be realized as hypersurfaces in a smooth projective variety?

Note that, in principle it could happen that there is a projective simple normal crossing variety that can be realized as a hypersurface in a complex manifold but not in a smooth projective variety.

Let $Y$ be a smooth variety and $D \subset Y$ a compact divisor. Let $D \subset$ $N \subset Y$ be a regular neighborhood with smooth boundary $\partial N$. If $D$ is the exceptional divisor of a resolution of an isolated singularity $x \in X$ 
then $\partial N$ is homeomorphic to the link $L(x \in X)$. It is clear that $D$ and $c_{1}\left(N_{D, X}\right) \in H^{2}(D, \mathbb{Z})$ determine the boundary $\partial N$, but I found it very hard to compute concrete examples.

Problem 70. Find an effective method to compute the cohomology or the fundamental group of $\partial N$, at least when $D$ is a simple normal crossing divisor.

\section{References}

$\left[\mathrm{ABC}^{+} 96\right]$ J. Amorós, M. Burger, K. Corlette, D. Kotschick, and D. Toledo, Fundamental groups of compact Kähler manifolds, Mathematical Surveys and Monographs, vol. 44, American Mathematical Society, Providence, RI, 1996. MR 1379330 (97d:32037)

[ABW09] D. Arapura, P. Bakhtary, and J. Włodarczyk, The combinatorial part of the cohomology of a singular variety, ArXiv:0902.4234, 2009.

[ABW11] Weights on cohomology, invariants of singularities, and dual complexes, ArXiv e-prints (2011).

[Art70] Michael Artin, Algebraization of formal moduli. II. Existence of modifications, Ann. of Math. (2) 91 (1970), 88-135. MR 0260747 (41 \#5370)

[Bar65] D. Barden, Simply connected five-manifolds, Ann. of Math. (2) 82 (1965), 365-385. MR MR0184241 (32 \#1714)

[Bea96] Arnaud Beauville, Complex algebraic surfaces, second ed., London Mathematical Society Student Texts, vol. 34, Cambridge University Press, Cambridge, 1996, Translated from the 1978 French original by R. Barlow, with assistance from N. I. Shepherd-Barron and M. Reid. MR MR97e:14045

[Ber02] A. J. Berrick, A topologist's view of perfect and acyclic groups, Invitations to geometry and topology, Oxf. Grad. Texts Math., vol. 7, Oxford Univ. Press, Oxford, 2002, pp. 1-28. MR 1967745 (2004c:20001)

[BG00] Charles P. Boyer and Krzysztof Galicki, On Sasakian-Einstein geometry, Internat. J. Math. 11 (2000), no. 7, 873-909. MR 2001k:53081

[BG08] _ Sasakian geometry, Oxford Mathematical Monographs, Oxford University Press, Oxford, 2008. MR 2382957 (2009c:53058)

[BGK05] Charles P. Boyer, Krzysztof Galicki, and János Kollár, Einstein metrics on spheres, Ann. of Math. (2) 162 (2005), no. 1, 557-580. MR MR2178969 (2006j:53058)

[BGKT05] Charles P. Boyer, Krzysztof Galicki, János Kollár, and Evan Thomas, Einstein metrics on exotic spheres in dimensions 7, 11, and 15, Experiment. Math. 14 (2005), no. 1, 59-64. MR 2146519 (2006a:53042)

[BM11] Edward Bierstone and Pierre D. Milman, Resolution except for minimal singularities I, arXiv.org:1107.5595, 2011.

[BP11] Edward Bierstone and Franklin V. Pacheco, Resolution of singularities of pairs preserving semi-simple normal crossings, arXiv.org:1109.3205, 2011.

[Bri66] Egbert Brieskorn, Beispiele zur Differentialtopologie von Singularitäten, Invent. Math. 2 (1966), 1-14. MR 34 \#6788

[Cai61] Stewart S. Cairns, A simple triangulation method for smooth manifolds, Bull. Amer. Math. Soc. 67 (1961), 389-390. MR 0149491 (26 \#6978)

[Cat91] Fabrizio Catanese, Moduli and classification of irregular Kaehler manifolds (and algebraic varieties) with Albanese general type fibrations, Invent. Math. 104 (1991), no. 2, 263-289. MR 1098610 (92f:32049)

[Cat96] _ Fundamental groups with few relations, Higher-dimensional complex varieties (Trento, 1994), de Gruyter, Berlin, 1996, pp. 163-165. MR 1463177 (98i:32047) 
[CMS08a] Sylvain E. Cappell, Laurentiu G. Maxim, and Julius L. Shaneson, Euler characteristics of algebraic varieties, Comm. Pure Appl. Math. 61 (2008), no. 3, 409-421. MR 2376847 (2009f:14038)

[CMS08b] Hodge genera of algebraic varieties. I, Comm. Pure Appl. Math. 61 (2008), no. 3, 422-449. MR 2376848 (2009f:14039)

[Coh73] Marshall M. Cohen, A course in simple-homotopy theory, Springer-Verlag, New York, 1973, Graduate Texts in Mathematics, Vol. 10. MR 0362320 (50 \#14762)

[Cor92] Jon Michael Corson, Complexes of groups, Proc. London Math. Soc. (3) 65 (1992), no. 1, 199-224. MR 1162493 (93h:57003)

[CS91] Sylvain E. Cappell and Julius L. Shaneson, Stratifiable maps and topological invariants, J. Amer. Math. Soc. 4 (1991), no. 3, 521-551. MR 1102578 (92d:57024)

[CS08] Kevin Corlette and Carlos Simpson, On the classification of rank-two representations of quasiprojective fundamental groups, Compos. Math. 144 (2008), no. 5, 1271-1331. MR 2457528 (2010i:14006)

[Dem88] Michel Demazure, Anneaux gradués normaux, Introduction à la théorie des singularités, II, Travaux en Cours, vol. 37, Hermann, Paris, 1988, pp. 35-68. MR 91k:14004

[dFKX12] Tommaso de Fernex, János Kollár, and Chenyang Xu, The dual complex of singularities, ArXiv e-prints (2012).

[DGMS75] Pierre Deligne, Phillip Griffiths, John Morgan, and Dennis Sullivan, Real homotopy theory of Kähler manifolds, Invent. Math. 29 (1975), no. 3, 245274. MR MR0382702 (52 \#3584)

[DH88] Alan H. Durfee and Richard M. Hain, Mixed Hodge structures on the homotopy of links, Math. Ann. 280 (1988), no. 1, 69-83. MR 928298 (89c:14012)

[Dol83] Igor V. Dolgachev, On the link space of a Gorenstein quasihomogeneous surface singularity, Math. Ann. 265 (1983), no. 4, 529-540. MR 721886 (85k:32024)

[DPS09] Alexandru Dimca, Stefan Papadima, and Alexander I. Suciu, Topology and geometry of cohomology jump loci, Duke Math. J. 148 (2009), no. 3, 405-457. MR 2527322 (2011b:14047)

[FM83] Robert Friedman and David R. Morrison (eds.), The birational geometry of degenerations, Progr. Math., vol. 29, Birkhäuser Boston, Mass., 1983. MR 690262 (84g:14032)

[Fuj09] Osamu Fujino, Introduction to the log minimal model program for log canonical pairs, arXiv.org:0907.1506, 2009.

[Fuj12a] Kento Fujita, Simple normal crossing Fano varieties and log Fano manifolds, ArXiv e-prints (2012).

[Fuj12b] The Mukai conjecture for log Fano manifolds, ArXiv e-prints (2012).

[FZ03] Hubert Flenner and Mikhail Zaidenberg, Normal affine surfaces with $\mathbb{C}^{*}$ actions, Osaka J. Math. 40 (2003), no. 4, 981-1009. MR 2020670

[GL91] Mark Green and Robert Lazarsfeld, Higher obstructions to deforming cohomology groups of line bundles, J. Amer. Math. Soc. 4 (1991), no. 1, 87-103. MR MR1076513 (92i:32021)

[GM88] Mark Goresky and Robert MacPherson, Stratified Morse theory, Ergebnisse der Mathematik und ihrer Grenzgebiete (3), vol. 14, Springer-Verlag, Berlin, 1988. MR 932724 (90d:57039)

[Gor80] Gerald Leonard Gordon, On a simplicial complex associated to the monodromy, Trans. Amer. Math. Soc. 261 (1980), no. 1, 93-101. MR 576865 (81j:32017)

[GR70] Hans Grauert and Oswald Riemenschneider, Verschwindungssätze für analytische Kohomologiegruppen auf komplexen Räumen, Invent. Math. 11 (1970), 263-292. MR MR0302938 (46 \#2081)

[Gro67] Alexander Grothendieck, Local cohomology, Lecture Notes in Mathematics, Vol. 41, Springer-Verlag, Berlin, 1967. MR 0224620 (37 \#219) 
[Gro68]__ Cohomologie locale des faisceaux cohérents et théorèmes de Lefschetz locaux et globaux ( $S G A$ 2), North-Holland Publishing Co., Amsterdam, 1968, Augmenté d'un exposé par Michèle Raynaud, Séminaire de Géométrie Algébrique du Bois-Marie, 1962, Advanced Studies in Pure Mathematics, Vol. 2. MR 0476737 (57 \#16294)

[Gro89] Michel Gromov, Sur le groupe fondamental d'une variété kählérienne, C. R. Acad. Sci. Paris Sér. I Math. 308 (1989), no. 3, 67-70. MR 983460 (90i:53090)

[GS75] Phillip Griffiths and Wilfried Schmid, Recent developments in Hodge theory: a discussion of techniques and results, Discrete subgroups of Lie groups and applicatons to moduli (Internat. Colloq., Bombay, 1973), Oxford Univ. Press, Bombay, 1975, pp. 31-127. MR 0419850 (54 \#7868)

[Har77] Robin Hartshorne, Algebraic geometry, Springer-Verlag, New York, 1977, Graduate Texts in Mathematics, No. 52. MR 0463157 (57 \#3116)

[Har95] Joe Harris, Algebraic geometry, Graduate Texts in Mathematics, vol. 133, Springer-Verlag, New York, 1995, A first course, Corrected reprint of the 1992 original. MR MR1416564 (97e:14001)

[Hat02] Allen Hatcher, Algebraic topology, Cambridge University Press, Cambridge, 2002. MR 1867354 (2002k:55001)

[Hig51] Graham Higman, A finitely generated infinite simple group, J. London Math. Soc. 26 (1951), 61-64. MR 0038348 (12,390c)

[Hir62] Morris W. Hirsch, Smooth regular neighborhoods, Ann. of Math. (2) 76 (1962), 524-530. MR 0149492 (26 \#6979)

[Ish85] Shihoko Ishii, On isolated Gorenstein singularities, Math. Ann. 270 (1985), no. 4, 541-554. MR MR776171 (86j:32024)

[Kap12] M. Kapovich, Dirichlet fundamental domains and complex-projective varieties, ArXiv e-prints (2012).

[Ker69] Michel A. Kervaire, Smooth homology spheres and their fundamental groups, Trans. Amer. Math. Soc. 144 (1969), 67-72. MR 0253347 (40 \#6562)

[KK11] Michael Kapovich and János Kollár, Fundamental groups of links of isolated singularities, Journal AMS (to appear) ArXiv e-prints (2011).

[KM63] Michel A. Kervaire and John W. Milnor, Groups of homotopy spheres. I, Ann. of Math. (2) 77 (1963), 504-537. MR 0148075 (26 \#5584)

[KM98a] Michael Kapovich and John J. Millson, On representation varieties of Artin groups, projective arrangements and the fundamental groups of smooth complex algebraic varieties, Inst. Hautes Études Sci. Publ. Math. (1998), no. 88, 5-95 (1999). MR 1733326 (2001d:14024)

[KM98b] János Kollár and Shigefumi Mori, Birational geometry of algebraic varieties, Cambridge Tracts in Mathematics, vol. 134, Cambridge University Press, Cambridge, 1998, With the collaboration of C. H. Clemens and A. Corti, Translated from the 1998 Japanese original. MR 1658959 (2000b:14018)

[Kob63] Shoshichi Kobayashi, Topology of positively pinched Kaehler manifolds, Tôhoku Math. J. (2) 15 (1963), 121-139. MR 0154235 (27 \#4185)

[Kol95] János Kollár, Shafarevich maps and automorphic forms, M. B. Porter Lectures, Princeton University Press, Princeton, NJ, 1995. MR 1341589 (96i:14016)

[Kol05] _ Einstein metrics on five-dimensional Seifert bundles, J. Geom. Anal. 15 (2005), no. 3, 445-476. MR MR2190241 (2007c:53056)

[Kol06] Circle actions on simply connected 5-manifolds, Topology 45 (2006), no. 3, 643-671. MR 2218760 (2006m:57044)

[Kol07a] Einstein metrics on connected sums of $S^{2} \times S^{3}$, J. Differential Geom. 75 (2007), no. 2, 259-272. MR MR2286822 (2007k:53061)

[Kol07b] L Lectures on resolution of singularities, Annals of Mathematics Studies, vol. 166, Princeton University Press, Princeton, NJ, 2007. MR 2289519 
[Kol09] _ Positive Sasakian structures on 5-manifolds, Riemannian topology and geometric structures on manifolds, Progr. Math., vol. 271, Birkhäuser Boston, Boston, MA, 2009, pp. 93-117. MR 2494170 (2010i:53077)

[Kol11] , New examples of terminal and log canonical singularities, arXiv:1107.2864, 2011.

[Kol12] _ Quotients by finite equivalence relations, Current developments in algebraic geometry, Math. Sci. Res. Inst. Publ., vol. 59, Cambridge Univ. Press, Cambridge, 2012, With an appendix by Claudiu Raicu, pp. 227-256. MR 2931872

[Kol13a] _ Simple normal crossing varieties with prescribed dual complex, ArXiv e-prints (2013).

[Kol13b] Singularities of the minimal model program, Cambridge University Press, Cambridge, 2013, With the collaboration of S. Kovács.

[Kul77] Vik. S. Kulikov, Degenerations of K3 surfaces and Enriques surfaces, Izv. Akad. Nauk SSSR Ser. Mat. 41 (1977), no. 5, 1008-1042, 1199. MR 0506296 (58 \#22087b)

[Mas61] W. S. Massey, Obstructions to the existence of almost complex structures, Bull. Amer. Math. Soc. 67 (1961), 559-564. MR 0133137 (24 \#A2971)

[Mor78] John W. Morgan, The algebraic topology of smooth algebraic varieties, Inst. Hautes Études Sci. Publ. Math. (1978), no. 48, 137-204. MR 516917 (80e:55020)

[Mum61] David Mumford, The topology of normal singularities of an algebraic surface and a criterion for simplicity, Inst. Hautes Études Sci. Publ. Math. (1961), no. 9, 5-22. MR 0153682 (27 \#3643)

[Neu81] Walter D. Neumann, A calculus for plumbing applied to the topology of complex surface singularities and degenerating complex curves, Trans. Amer. Math. Soc. 268 (1981), no. 2, 299-344. MR 632532 (84a:32015)

[OW75] Peter Orlik and Philip Wagreich, Seifert n-manifolds, Invent. Math. 28 (1975), 137-159. MR 50 \#13596

[Pay09] Sam Payne, Lecture at MSRI, http://www.msri.org/web/msri/online-videos//video/showVideo/3674, 2009.

[Pay11] Sam Payne, Boundary complexes and weight filtrations, ArXiv e-prints (2011).

[Per77] Ulf Persson, On degenerations of algebraic surfaces, Mem. Amer. Math. Soc. 11 (1977), no. 189, xv+144. MR 0466149 (57 \#6030)

[Pin77] H. Pinkham, Normal surface singularities with $C^{*}$ action, Math. Ann. 227 (1977), no. 2, 183-193. MR 55 \#5623

[PP08] Patrick Popescu-Pampu, On the cohomology rings of holomorphically fillable manifolds, Singularities II, Contemp. Math., vol. 475, Amer. Math. Soc., Providence, RI, 2008, pp. 169-188. MR 2454366 (2010h:32039)

[PS08] Chris A. M. Peters and Joseph H. M. Steenbrink, Mixed Hodge structures, Ergebnisse der Mathematik und ihrer Grenzgebiete. 3. Folge., vol. 52, SpringerVerlag, Berlin, 2008. MR MR2393625

[Rez02] Alexander Reznikov, The structure of Kähler groups. I. Second cohomology, Motives, polylogarithms and Hodge theory, Part II (Irvine, CA, 1998), Int. Press Lect. Ser., vol. 3, Int. Press, Somerville, MA, 2002, pp. 717-730. MR 1978716 (2004c:32042)

[Sco83] Peter Scott, The geometries of 3-manifolds, Bull. London Math. Soc. 15 (1983), no. 5, 401-487. MR 84m:57009

[Sei32] Herbert Seifert, Topologie dreidimensionaler gefaserte Räume, Acta Math. 60 (1932), 148-238.

[Ser77] Jean-Pierre Serre, Arbres, amalgames, $\mathrm{SL}_{2}$, Société Mathématique de France, Paris, 1977, Avec un sommaire anglais, Rédigé avec la collaboration de Hyman Bass, Astérisque, No. 46. MR 0476875 (57 \#16426) 
[Sim10] Carlos Simpson, Local systems on proper algebraic V-manifolds, arXiv1010.3363, 2010.

[Siu87] Yum Tong Siu, Strong rigidity for Kähler manifolds and the construction of bounded holomorphic functions, Discrete groups in geometry and analysis (New Haven, Conn., 1984), Progr. Math., vol. 67, Birkhäuser Boston, Boston, MA, 1987, pp. 124-151. MR 900825 (89i:32044)

[Sma62] Stephen Smale, On the structure of 5-manifolds, Ann. of Math. (2) 75 (1962), 38-46. MR 25 \#4544

[Ste83] J. H. M. Steenbrink, Mixed Hodge structures associated with isolated singularities, Singularities, Part 2 (Arcata, Calif., 1981), Proc. Sympos. Pure Math., vol. 40, Amer. Math. Soc., Providence, RI, 1983, pp. 513-536. MR 713277 (85d:32044)

[Ste08] D. A. Stepanov, A note on resolution of rational and hypersurface singularities, Proc. Amer. Math. Soc. 136 (2008), no. 8, 2647-2654. MR 2399025 (2009g:32060)

[Sul77] Dennis Sullivan, Infinitesimal computations in topology, Inst. Hautes Études Sci. Publ. Math. (1977), no. 47, 269-331 (1978). MR 0646078 (58 \#31119)

[Thu07] Amaury Thuillier, Géométrie toroïdale et géométrie analytique non archimédienne. Application au type d'homotopie de certains schémas formels, Manuscripta Math. 123 (2007), no. 4, 381-451. MR 2320738 (2008g:14038)

Princeton University, Princeton, NJ 08544-1000, USA

E-mail address: kollar@math.princeton.edu 